\title{
Multidimensional hydrodynamical simulations of radiative cooling SNRs-clouds interactions: an application to starburst environments
}

\author{
C. Melioli ${ }^{1}$, E. M. de Gouveia Dal Pino ${ }^{1}$, and A. Raga ${ }^{2}$ \\ 1 Universidade de São Paulo, IAG, Rua do Matão 1226, Cidade Universitária, São Paulo 05508-900, Brazil \\ e-mail: [cmelioli; dalpino]@astro.iag.usp.br \\ 2 Instituto de Ciencias Nucleares, Universidad Nacional Autónoma de México, Apartado Postal 70-543, 04510 México D.F., México
}

Received 11 January 2005 / Accepted 18 July 2005

ABSTRACT

\begin{abstract}
Most galaxies present supernova shock fronts interacting with cloudy interstellar medium. These interactions can occur either at small scales, between a single supernova remnant (SNR) and a compact cloud, or at large scales, between a giant shell of a superbubble and a molecular cloud. Here study the by-products of SNR-clouds in a starburst (SB) system. Due to the high supernova (SN) rate in this environment, a cloud may be shocked more than once by SNRs. These interactions can have an important role in the recycling of matter from the clouds to the ISM and vice-versa. Their study is also relevant to understand the evolution of the ISM density and the structure of the clouds embedded in it.

In the present work, we have focused our attention on the global effects of the interactions between clouds and SN shock waves in the ISM of SB environments and performed three-dimensional radiative cooling hydrodynamical simulations which have included the continuity equations for several atomic/ionic and molecular species. We have also considered the effects of the photo-evaporation due to the presence of the UV radiation from hot stars and $\mathrm{SNe}$.

The results have shown that due to the presence of radiative cooling, the interactions cause the formation of elongated cold filaments, instead of favoring an efficient mixing of the cloud gas with the diffuse ISM. These filaments could be associated with the dense clumps observed inside several SBs that are blown out by the galactic wind. The results have also revealed that the SNR-cloud interactions are less efficient at producing substantial mass loss from the clouds to the diffuse ISM than mechanisms such as the photo-evaporation caused by the UV flux from the hot stars. This result has important consequences for the global evolution of the SB environment and the formation of the associated superwinds, and may also be relevant to the ISM of normal galaxies.
\end{abstract}

Key words. galaxies: starburst - hydrodynamics - shock waves - methods: N-body simulations - ISM: clouds ISM: supernova remnants

\section{Introduction}

Starburst (SB) systems are gas-rich environments where, due to a variety of phenomena, the fraction of gas mass that is converted into stars, $\epsilon$, is $\sim 10$ times higher than in normal galaxies (Colina et al. 1991). In some extreme cases, the star formation rate (SFR) can be of the order of $100 M_{\odot} \mathrm{yr}^{-1}$ (Scoville \& Soifer 1990), while in galaxies such as M 82, one of the most studied SB galaxies, it is $\sim 5 M_{\odot} \mathrm{yr}^{-1}$. Due to the high number of massive stars, these systems present high rates of supernova ( $\mathrm{SN}$ ) explosions. Also, the presence of $\mathrm{O}$ and $\mathrm{B}$ stars is expected to produce large UV photons fluxes that can photoionize and evaporate compact structures embedded in the interstellar medium (ISM) (Bertoldi 1989; Bertoldi \& McKee 1990; Gorti \& Hollenbach 2002).

Shock fronts of SN remnants (SNRs) collide with each other and with the clouds of the ISM, and this can lead to gas mixing and recycling of matter from the clouds to the ISM and vice-versa. Chandra observations of SB regions have revealed the presence of expanding supershells (e.g. Ott et al. 2003) due to multiple SN explosions and strong stellar winds, therefore suggesting that interactions between shock fronts and inhomogeneities of the ambient medium can occur either at small scales, as for example, between a single SNR and a compact cloud or globule, or at large scales, like the encounter of a giant shell of a superbubble with a molecular cloud.

Several authors have studied shock wave-cloud interactions using both analytical and numerical approaches (e.g. Hartquist et al. 1986; Klein et al. 1994; Anderson et al. 1994; Jun et al. 1996; Redman et al. 1998; Jun \& Jones 1999; Lim \& Raga 1999; de Gouveia Dal Pino 1999; Poludnenko et al. 2002, hereafter PFB02; Fragile et al. 2004; Steffen \& Lopez 2004; Fragile et al. 2005; Marcolini et al. 2005), to understand the dynamics of the propagation of the shock front into a cloud, cloud mass loading, changes in the chemical composition, and the dependence of these processes on the parameters of the system, such 
as the shell and the cloud densities, the ambient temperature and the shell velocity. In general, these studies are focused on a single clump and only explore the processes of mass loading and the physical evolution of the shocked gas in the cloud. Exceptions are the studies of Steffen \& Lopez (2004), which investigate the interaction of a wind with several clumps, and the studies of Jun et al. (1996) and, more recently, the studies of PFB02 and Fragile et al. (2004), which focus on the interstellar gas enrichment that may result from the interaction of a planar shock wave with cylindrical clouds. Klein et al. (1994), for instance, have shown that a cloud is destroyed in a time interval of the order of a few times the cloud crushing time, that is, the time needed for the internal forward shock to cross the cloud and reach its downstream surface. This result is confirmed by PFB02, and thus an efficient gas mixing is expected after the interaction of a shock wave with inhomogeneous ISM. However, these studies have been performed for adiabatic flows in a twodimensional space. Since the typical timescales for radiative cooling in SBs $\left(10^{4}-10^{5} \mathrm{yr}\right)$ are smaller than their dynamical timescales, the radiative losses may have important effects on the dynamical evolution of these systems and therefore an adiabatic treatment of the flow is not completely appropriate.

Recently, Melioli \& de Gouveia Dal Pino (2004) have carried out a study to estimate the gas density evolution in SB galaxies. In such environments the gas exchange between the clouds and the diffuse ISM can be very fast, mainly due to efficient thermal and photo-evaporation of the clouds on short time scales compared with the dynamical time of the SB. However, these analytical studies have described only approximately the also important but rather complex interactions between these clouds and the shock fronts of the SNe. In the present study we try to explore the mass loss evolution of the clouds embedded in the ISM of a SB with the help of fully three-dimensional gas dynamical simulations. Employing a modified version of the YGUAZU code (Raga et al. 2000; Raga et al. 2002; Masciadri et al. 2002) which also includes the effects of gas radiative cooling and a photoionizing flux of UV photons due to OB stars, we investigate the evolution of the clouds that are shocked by SN blast waves. Though mainly focusing on the conditions of a SB environment, the present study also has applications to the ISM of normal galaxies.

We notice that a cold cloud embedded in a hot medium could also evaporate at high rates due to thermal conduction effects (Begelman \& McKee 1990; McKee \& Begelman 1990). However, the clouds assumed in our study must evaporate in a time $\sim 10^{6}$ yr (Melioli \& de Gouveia Dal Pino 2004) which is much larger than the timescales examined here (see below) and therefore we ignored its effects. For a detailed study of a wind-cloud interaction including the effects of the thermal conduction, we refer to the recent work of Marcolini et al. (2005). We have also not considered the presence of magnetic fields embedded in the clouds and the environment. As remarked in Melioli \& de Gouveia Dal Pino (2004), they would provide extra support to clouds against destruction in the interactions and would also inhibit the effects of the thermal conduction in the direction normal to their dominating component. (Magnetic field effects will be examined in future study.)
In Sect. 2 we discuss the numerical technique employed in the YGUAZU code and the input conditions of the problem. The results of the simulations are presented in Sect. 3; in Sect. 4 we discuss the results, then compare them with those obtained by PFB02 and outline the conclusions. Some consequences for the evolution of the ISM in SB galaxies are also outlined.

\section{Numerical simulations}

\subsection{The numerical method}

To simulate the cloud-SNR interactions we have used a modified version of the numerical adaptive mesh refinement YGUAZUa hydrodynamical code (see Raga et al. 2000; Raga et al. 2002) without including UV flux, and the YGUAZUb code (Raga \& Reipurth 2004) that considers UV flux. The YGUAZUa code integrates the gas-dynamic equations with the "flux vector splitting" algorithm of Van Leer (1982) and solves a system of rate equations for atomic/ionic and molecular species. It also includes a parameterized cooling function in the energy equation that allows the gas to cool from $\sim 10^{6}$ to $1000 \mathrm{~K}$ with errors smaller than $10 \%$. In order to integrate the system of atomic/ionic and molecular rate equations, a semi-implicit method by Young \& Boris (1973) is used. The YGUAZUb code includes the computation of the transfer of a direct ionizing photon flux (at the Lyman limit), and the diffuse ionizing field is considered by taking only the case B recombination for the hydrogen.

The 3-D adaptive, binary, hierarchical computational grid, in which the gas-dynamic equations are integrated, is structured with a base grid, and with higher resolution grids. The lower resolution grids which correspond to $n=1$ and $n=2$, are defined over the whole computational domain. The higher resolution grids are defined in smaller regions of the domain and corresponds to $n=3,4, \ldots, n_{\max }$, with successive increase in resolution by factors of 2 . In this study we have adopted $n_{\max }=4$.

\subsection{Description of the problem and the computational domain}

Our simulations are developed in a three-dimensional computational domain that mimics the ISM of a galaxy with the diffuse gas characterized by an initial density $\rho_{\mathrm{a}}$, and a temperature $T_{\mathrm{a}}$, and with embedded clouds with initial density $\rho_{\mathrm{c}}$, and temperature $T_{\mathrm{c}}$. These values determine the density contrast, $\chi=\rho_{\mathrm{c}} / \rho_{\mathrm{a}}$, and the sound speed, $C_{\mathrm{s}}^{2}=\gamma k T_{\mathrm{a}} / \mu m_{\mathrm{H}}$, where $k$ is the Boltzman constant of the gas, $\mu$ is the mean mass per particle of the gas, and $\gamma$ is the ratio between the specific heats at constant volume and the pressure, respectively. In this work, we take $\mu_{i}=0.63$ for the ionized gas, and $\mu_{n}=1.3$ for the neutral gas, assuming a gas with $90 \% \mathrm{H}$ and $10 \% \mathrm{He}$ abundances, and $\gamma=5 / 3$.

A shock wave front is allowed to propagate into this environment from the bottom to the top of the box with a velocity $v_{\mathrm{sh}}=7 C_{\mathrm{s}}$, so that the Mach number is $M_{\mathrm{s}}=7$. If the shock front interacts with the clouds on a timescale shorter than the cooling time of the shocked material, then the shock front can be assumed to be non-radiative. However, we will 
see below that this condition is not always fulfilled. From the Rankine-Hugoniot relations, we find:

$\rho_{\text {sh }}=\frac{2.67 M_{\mathrm{s}}^{2}}{2+0.67 M_{\mathrm{s}}^{2}} \rho_{\mathrm{g}}$

$T_{\mathrm{sh}}=\left(1.25 M_{\mathrm{s}}^{2}-0.25\right) \frac{\rho_{\mathrm{g}} T_{\mathrm{g}}}{\rho_{\mathrm{sh}}}$

$p_{\mathrm{sh}}=M_{\mathrm{s}}^{2} p_{\mathrm{g}}$

where $\rho_{\mathrm{sh}}, T_{\mathrm{sh}}$ and $p_{\mathrm{sh}}$ are the density, the temperature and the pressure behind the shock front, respectively, and $p_{\mathrm{g}}$ is the pressure of the diffuse gas.

To resolve the physical conditions of the clouds, Klein et al. (1994) have suggested that a minimum resolution of 120 cells per cloud is necessary, but PFB02 have shown that between a maximum and a minimum resolution of 120 and 32 cells per radius, respectively, the only difference is the rate at which the instabilities develop, and this does not seem to have an important effect on the global evolution of the system. In this study, the highest resolution grid that covers the computational volume has 1024 cells along the $Z$ direction, 512 cells along the $X$ direction and 256 cells along the $Y$ direction. This corresponds to physical dimensions for the box of $0.8 \times 0.4 \times 1.6 \mathrm{pc}$, respectively. We notice that in some of the models to be described below, where an otherwise unrealistically high cpu time would have prevented us from obtaining results within the relevant physical times of the interactions, we have adopted only 32 cells along the $Y$ direction instead of 256 . In these cases, the numerical tests can be considered nearly 2-D, as the computational domain has an extension of only one cloud diameter in this direction, and the main differences with regard to previous 2-D investigations are the geometry of the clouds and the presence of radiative cooling (see below).

All the clouds are assumed to be spherical with a physical radius of $0.05 \mathrm{pc}$. The cloud diameter corresponds to $1 / 8$ of the $X$-box extension and $1 / 16$ of the $Z$-box extension. In the $Y$ direction the box is assumed to be 2 or 8 times the cloud radius. With these dimensions, the radius of each cloud occupies 32 cells, as in the adiabatic study of PFB02. Figure 1 illustrates the computational setup described above. We notice that numerical tests made with a cloud occupying a number of cells that is twice as large have produced similar results although revealing more details in the overall filamentary structure that develops later on in the cloud, due to the higher resolution. This is to be expected, particularly in radiative cooling interactions where a combination of non-uniform radiative cooling at the shocked accelerated regions may trigger the development of Rayleigh-Taylor instabilities that tend to enrich the formation of substructures, like in the shock fronts of supernova explosions and protostellar jets (see e.g., Blondin et al. 1990; de Gouveia Dal Pino \& Benz 1993; see also the discussion in Sect. 3.1). However these substructures also appear in the lower resolution simulations. On the other hand, Kelvin-Helmholtz (K-H) instabilities, whose development would favor fragmentation due to shear at the contact discontinuity between the clouds and the wind, are expected to be less efficient in the presence of radiative cooling. This tends to produce a colder

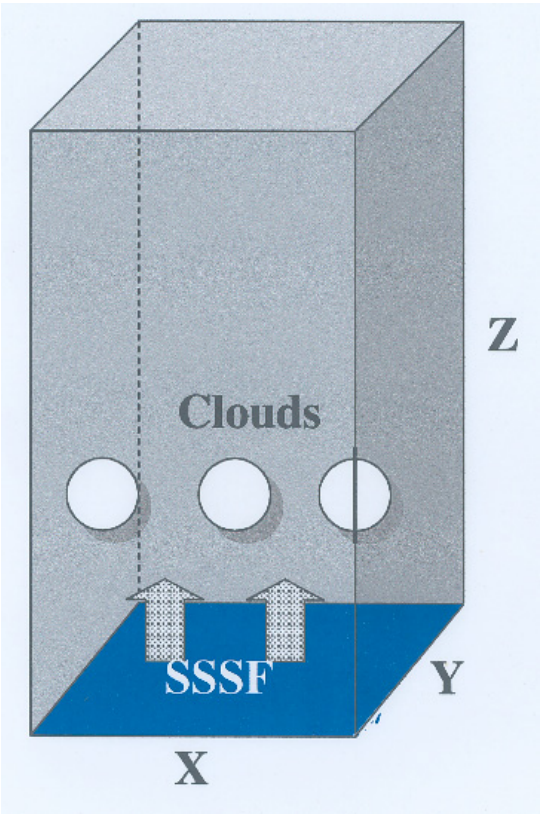

Fig. 1. Setup of the computational domain (not in scale). The SSSF is injected through the bottom boundary of the box. The boundaries are transmitting on all the faces of the box.

and lower pressure gas which will be less efficient in triggering oblique shocks and the growth of K-H unstable modes than in adiabatic interactions (e.g., de Gouveia Dal Pino \& Benz 1993; Stone et al. 1997; Cerqueira \& de Gouveia Dal Pino 1999). Altogether these points suggest that the adoption in the present study of a grid with similar resolution to that employed in earlier adiabatic studies (PFB02, Fragile et al. 2004) will not result in the loss of relevant information on the development of structures and fragmentation in the SNR-cloud interaction.

For a better comprehension and interpretation of the SNR-cloud interactions, we will introduce four physical parameters that are able to describe the evolution of the system. As in PFB02, we define the shock-crossing time as the time necessary for the incident shock wave to sweep a single cloud:

$t_{\mathrm{SC}}=\frac{2 r_{\mathrm{c}}}{v_{\mathrm{sh}}}$

where $r_{\mathrm{c}}$ is the cloud radius and $v_{\mathrm{sh}}$ is the shell velocity. Hereafter, all the time intervals will be defined as a function of the shock-crossing time. We note that radiative cooling simulations are not scale-independent. In other words, systems with different physical dimensions will result a distinct behavior if radiative cooling is present. For this reason, $t_{\mathrm{SC}}$ is not the only parameter that controls the evolutionary status of the interactions. However, $t_{\mathrm{SC}}$ is still the best characteristic time scale to compare the results of simulations with different initial conditions.

We also define the normalized cloud velocity, $v_{\mathrm{c}, \mathrm{N}}$, as the ratio between the velocity of the gas in the cloud core and the initial velocity of the shock wave:

$v_{\mathrm{c}, \mathrm{N}}=\frac{v_{\mathrm{c}, t}}{v_{\mathrm{sh}}}$

where $v_{\mathrm{c}, t}$ is the cloud velocity at time $t$. 
Another useful parameter is the normalized mass loss, defined as the ratio between the total mass loss of the clouds at a time $t\left(M_{1, t}\right.$, in solar mass) and the initial total mass of the clouds:

$M_{1, n}=\frac{M_{1, t}}{m_{\mathrm{c}} N_{\mathrm{c}}}$

where $N_{\mathrm{c}}$ is the number of clouds per simulation and $m_{\mathrm{c}}$ the initial mass of each cloud. This parameter gives a measure of the effective ablation of the cloud after the passage of a shock front at a time $t$.

We define a typical timescale for the evolution of the cloud density. We may trace its evolution by defining a timescale $\tau_{\mathrm{c}}$ such that $\rho_{\mathrm{c}}(t)=\rho_{\mathrm{c}} e^{-t / \tau_{\mathrm{c}}}$, and $\tau_{\mathrm{c}}$ corresponds to the time necessary to reduce the density of the cloud by $1 / e$. We will assume that all the gas having a density greater than $\rho_{\mathrm{c}} / e$ is still part of the cloud. This assumption will allow us to distinguish the cloud core from the ablated gas that may eventually mix with the diffuse ISM (see below) and therefore, to compute the mass loss.

Finally, we define the fragmentation factor as the number of fragments, $\mathcal{N}_{\mathrm{f}}$, per cloud, at a time $t$ :

$f_{\mathrm{f}, t}=\frac{\mathcal{N}_{\mathrm{f}}}{N_{\mathrm{c}}}$

where we will still consider a "cloud fragment" as all the gas with density greater than $1 / e$ of the initial cloud density embedded in a more tenuous gas.

\subsection{Input conditions}

We have performed several simulations considering both adiabatic and radiative cooling systems. In a first set, we have considered interactions between a steady state shock front (SSSF) and a group of clouds in the absence of a flux of UV photons. We have assumed an initially ionized ambient medium with a mass density $\rho_{\mathrm{a}}=2.15 \times 10^{-25} \mathrm{~g} \mathrm{~cm}^{-3}$, and a temperature $T_{\mathrm{a}}=$ $10^{4} \mathrm{~K}$. Clouds with mass density $\rho_{\mathrm{c}}=1.075 \times 10^{-22} \mathrm{~g} \mathrm{~cm}^{-3}$ and temperature $T_{\mathrm{c}}=100 \mathrm{~K}$ are embedded in the ambient medium. We notice that although the clouds and the ambient medium are initially not in perfect thermal pressure equilibrium, this condition will have no relevant effect on the interaction between the clouds and the SSSF since the cloud sound-wave crossing time is longer than the dynamical timescales of the interactions. The ratio $\chi$ between the cloud and the ambient density is 500 . Using Eqs. (1) to (3), we inject an SSSF with mass density $\rho_{\text {sh }}=8.6 \times 10^{-25} \mathrm{~cm}^{-3}$ and temperature $T_{\text {sh }}=1.5 \times 10^{5} \mathrm{~K}$ with a velocity of $104 \mathrm{~km} \mathrm{~s}^{-1}$. In this set of simulations, only the first run (SA1) involves an adiabatic interaction. The other simulations involve radiative-cooling interactions of an SSSF with one (SR1), two (SR2) and three clouds (SR3).

In the second set we have performed simulations with the same initial conditions of the first set, but assuming the presence of a flux of UV photons which allows at least partial photo-evaporation of the clouds. Interactions between an SSSF with one (SRP1), two (SRP2) and three clouds (SRP3) are also considered.
Finally, in a third set of simulations, an SNR shell (instead of a steady state shock front) interacts with one and two clouds without the presence of UV photons (SNS1 and SNS2, respectively), and also in the presence of a flux of UV photons (SNSP1 and SNSP2, respectively). In this set, the physical structure of the SNR is similar to that described in the works of Chevelier (1974) and Cioffi \& Shull (1992). The density and the temperature of the ambient gas were chosen to reproduce the typical conditions of a SB environment filled by a superbubble, with $\rho_{\mathrm{a}}=2.15 \times 10^{-26} \mathrm{~g} \mathrm{~cm}^{-3}$ and $T=10^{4} \mathrm{~K}$. In the cases without photo-evaporation, we have assumed an initial density contrast between the clouds and the ambient medium $\chi=100$, for it is more appropriate to a SB system, while in the cases with photo-evaporation, the clouds have a much higher pressure than the ISM and the $\chi$ parameter is equal to 5000 (see, e.g., Bertoldi \& McKee 1990). The SNR shell is injected with a velocity of $250 \mathrm{~km} \mathrm{~s}^{-1}$ and is assumed to have a thickness $h_{\mathrm{sh}}=1 \mathrm{pc}$, a density $\rho_{\mathrm{sh}}=8.6 \times 10^{-26} \mathrm{~g} \mathrm{~cm}^{-3}$ and a temperature $T_{\mathrm{sh}}=8.2 \times 10^{5} \mathrm{~K}$.

A summary of the parameters used in the three sets of simulations is shown in Table 1. The simulations with the computational domain extension in the $Y$ direction corresponding to twice the radius of the clouds have been labelled as 2-D to distinguish from those (labelled 3-D) at which the box extension in this direction was taken to be four times the cloud radius.

\section{Results}

\subsection{Set 1}

The first set of simulations presents the interactions between clouds and a SSSF without considering UV photon flux (runs SA1, SR1, SR2, SR3).

When an adiabatic cloud is impacted, it passes through four characteristic phases: compression, re-expansion, fragmentation and mixing with the ISM (see, e.g., Nittmann et al. 1982; McKee 1988; Klein et al. 1994). Its gas is initially compressed by an internal forward shock wave that crosses the cloud in a time $t_{\text {crush }} \sim t_{\mathrm{SC}} q^{0.5}$, where $q=\rho_{\mathrm{c}} / \rho_{\mathrm{sh}}$ is the ratio between the density of the cloud and the SSSF. For the models of the Set 1 , $q=125^{1}$. At the same time, a bow shock forms around the cloud and a reverse shock is generated downstream behind the cloud. This shock is caused by the convergence of the global flow behind the cloud and propagates with a velocity lower than the forward internal shock. It is dissipated very fast, compared with the shock-crushing time, and may be considered as a subsonic wave unable to change substantially the physical conditions of the cloud. The characteristics of the initial compression phase are well summarized in Fig. 2, where we present the results of the run SA1 for a non-radiative interaction between an SSSF and a cloud. This result is similar to the one obtained by PFB02 for similar initial conditions.

As in PFB02, we can distinguish the forward (A), the reverse (B) and the bow shock (C), the back flow (D), the primary and secondary vortex sheets $(\mathrm{E}, \mathrm{F})$ and the Mach reflected

\footnotetext{
${ }^{1}$ Our value of the density contrast between the cloud and the SSSF, $q$, differs from that in the simulations of PFB02 by a factor of 4 .
} 
Table 1. Parameters assumed in the numerical simulations of interactions between clouds and an SSSF or an SNR [(1): yr; (2): pc; (3): $2.15 \times 10^{-24} \mathrm{~g} \mathrm{~cm}^{-3}$; (4): $\mathrm{K} ;(5): \mathrm{km} \mathrm{s}^{-1}$ ].

\begin{tabular}{cccccccccccccccc}
\hline \hline Run & $t_{\mathrm{sC}}^{(1)}$ & $\mathrm{UV}$ & Cells & Geometry & $r_{\mathrm{c}}^{(2)}$ & $\rho_{\mathrm{a}}^{(3)}$ & $T^{(4)}$ & $\rho_{\mathrm{c}}^{(3)}$ & $T_{\mathrm{c}}^{(4)}$ & $N_{\mathrm{c}}$ & $\rho_{\mathrm{sh}}^{(3)}$ & $T_{\mathrm{sh}}{ }^{(4)}$ & $v_{\mathrm{sh}}{ }^{(5)}$ & $h_{\mathrm{sh}}^{(2)}$ & $M$ \\
\hline SA1 & 940 & $\mathrm{~N}$ & 32 & 2D & 0.05 & 0.1 & $10^{4}$ & 50 & 100 & 1 & 0.4 & $1.5 \times 10^{5}$ & 104 & $\infty$ & 7 \\
SR1 & 940 & $\mathrm{~N}$ & 32 & 3D & 0.05 & 0.1 & $10^{4}$ & 50 & 100 & 1 & 0.4 & $1.5 \times 10^{5}$ & 104 & $\infty$ & 7 \\
SR2 & 940 & $\mathrm{~N}$ & 32 & 3D & 0.05 & 0.1 & $10^{4}$ & 50 & 100 & 2 & 0.4 & $1.5 \times 10^{5}$ & 104 & $\infty$ & 7 \\
SR3 & 940 & $\mathrm{~N}$ & 32 & 3D & 0.05 & 0.1 & $10^{4}$ & 50 & 100 & 3 & 0.4 & $1.5 \times 10^{5}$ & 104 & $\infty$ & 7 \\
\hline SRP1 & 940 & $\mathrm{Y}$ & 32 & 3D & 0.05 & 0.1 & $10^{4}$ & 50 & 100 & 1 & 0.4 & $1.5 \times 10^{5}$ & 104 & $\infty$ & 7 \\
SRP2 & 940 & $\mathrm{Y}$ & 32 & 2D & 0.05 & 0.1 & $10^{4}$ & 50 & 100 & 2 & 0.4 & $1.5 \times 10^{5}$ & 104 & $\infty$ & 7 \\
\hline SNS1 & 391 & $\mathrm{~N}$ & 32 & 2D & 0.05 & 0.01 & $10^{4}$ & 1 & 100 & 1 & 0.04 & $8.2 \times 10^{5}$ & 250 & 1 & 17 \\
SNS2 & 391 & $\mathrm{~N}$ & 32 & 2D & 0.05 & 0.01 & $10^{4}$ & 1 & 100 & 2 & 0.04 & $8.2 \times 10^{5}$ & 250 & 1 & 17 \\
SNSP1 & 391 & $\mathrm{Y}$ & 32 & 3D & 0.05 & 0.01 & $10^{4}$ & 50 & 100 & 1 & 0.04 & $8.2 \times 10^{5}$ & 250 & 1 & 17 \\
SNSP2 & 391 & $\mathrm{Y}$ & 32 & 2D & 0.05 & 0.01 & $10^{4}$ & 50 & 100 & 2 & 0.04 & $8.2 \times 10^{5}$ & 250 & 1 & 17 \\
\hline
\end{tabular}

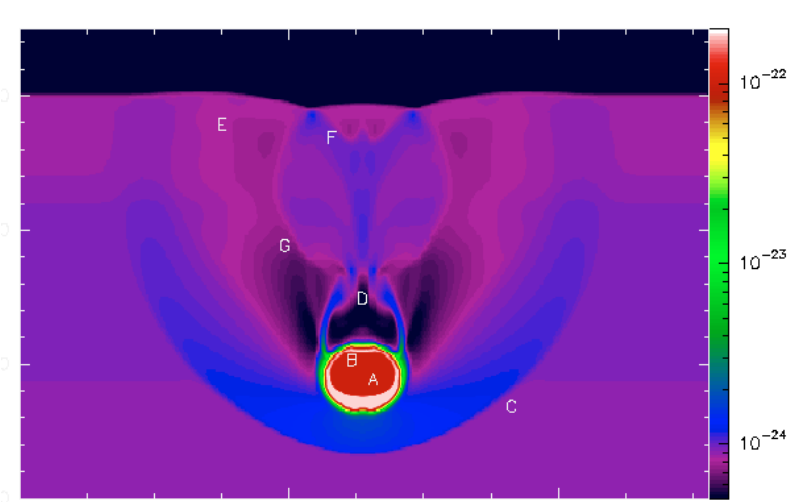

Fig. 2. Initial compression phase at a time $t=3 t_{\mathrm{SC}}$. Color-scale map of the midplane density distribution (in log scale) for an adiabatic (or non-radiative cooling) interaction between an SSSF and a cloud (model SA1 of Table 1). The density is in $\mathrm{g} \mathrm{cm}^{-3}$.

shocks $(\mathrm{G})^{2}$. A detailed description of these structures is also presented by, e.g., Klein et al. (1994) and PFB02. The comparison of the density distribution of the system at two different epochs (Fig. 3) with the non-radiative numerical simulations of PFB02 reveals the close similarity between both results, and therefore confirms the previous calculations.

We note that after a compression phase, the cloud starts to expand over the ambient medium. The sideways expansion of the cloud and the relative motion between it and the SSSF excites the development of oblique shocks and the Kelvin-Helmholtz instability at the cloud edge that causes some entrainment of the ambient material into the cloud. Also, the frontal acceleration of the much denser cloud material on the more rarefied ambient gas makes the system become Rayleigh-Taylor unstable and this causes the development of filamentary structure or the umbrella-type shape of the cloud seen in Fig. 3. The evolution of these instabilities causes a mixing of the cloud gas with the ISM. Similar effects have been

\footnotetext{
2 The back flow is caused by the global forward shock convergence on the symmetry axis; the Mach reflected shocks are caused by Mach reflection of the global forward shock at the symmetry shock; the primary and secondary vortex sheets are caused by regular reflection of the bow shock and by the primary Mach reflection of the forward shock, respectively (PFB02).
}

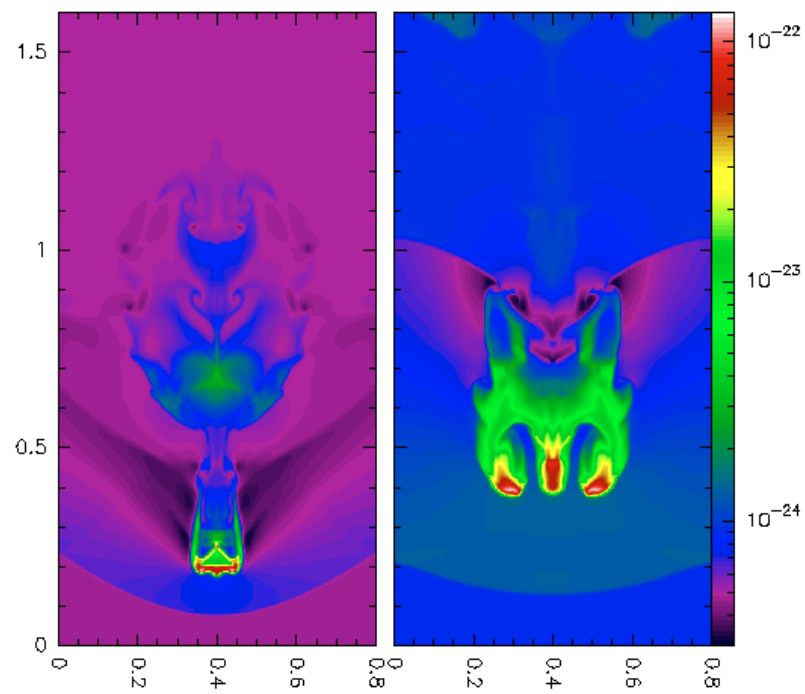

Fig. 3. Color-scale map of the midplane density distribution (in log scale) for the non-radiative interaction of a SSSF with a cloud with $m_{\mathrm{c}} \sim 0.001 M_{\odot}, r_{\mathrm{c}}=0.05 \mathrm{pc}$, and $\rho_{\mathrm{c}}=1.075 \times 10^{-22} \mathrm{~cm}^{-3}$ (model SA1 of Table 1). The shell has a density $\rho_{\mathrm{sh}}=8.6 \times 10^{-25} \mathrm{~g} \mathrm{~cm}^{-3}$ and velocity of $104 \mathrm{~km} \mathrm{~s}^{-1}$, and the ambient medium has $\rho_{\mathrm{a}}=2.15 \times$ $10^{-25} \mathrm{~g} \mathrm{~cm}^{-3}$ and $T=10^{4} \mathrm{~K}$. The computational box has dimensions $0.8 \mathrm{pc} \times 0.8 \mathrm{pc} \times 1.6 \mathrm{pc}$, corresponding to $512 \times 512 \times 1024$ grid points at the highest grid level. Time steps are $t=22 t_{\mathrm{SC}} \mathbf{a}$ ), and $t=36 t_{\mathrm{SC}} \mathbf{b}$ ). The density is shown in units of $\mathrm{g} \mathrm{cm}^{-3}$.

also found by PFB02 (see snapshots $t=50.54 t_{\mathrm{SC}}$ and $68.40 t_{\mathrm{SC}}$ of their Fig. 2).

On the other hand, when radiative cooling is considered, we expect that the mixing with the interstellar gas is delayed, and this can be seen by comparing the timescales for the setup of the Rayleigh-Taylor (R-T) and Kelvin-Helmholtz (K-H) instabilities with the timescale for radiative cooling.

The growth of the R-T instability due to the acceleration of the cloud by the postshock background gas is (e.g., Fragile et al. 2004):

$\tau_{\mathrm{R}-\mathrm{T}} \sim r_{\mathrm{c}} \frac{q^{0.5}}{\left(k r_{\mathrm{c}}\right)^{0.5} v_{\mathrm{sh}}}$

where $k$ is the wavenumber of the unstable mode. For $q \gg 1$ the timescale for growth of the K-H instability is $t=q^{0.5} /\left(k v_{\text {rel }}\right)$ 
(Chandrasekhar 1961), where $v_{\text {rel }}$ is the relative velocity between the postshock background and the cloud. Since the cloud accelerates rather slowly, the relative velocity is approximately the same as the postshock velocity, and thus

$$
\tau_{\mathrm{K}-\mathrm{H}} \sim r_{\mathrm{c}} \frac{q^{0.5}}{\left(k r_{\mathrm{c}}\right) v_{\mathrm{sh}}}
$$

Wavelengths corresponding to $k r_{\mathrm{c}}=1$ are the most disruptive, and thus $2 \tau_{\mathrm{R}-\mathrm{T}} \sim 2 \tau_{\mathrm{K}-\mathrm{H}} \sim t_{\mathrm{SC}} q^{0.5} \simeq 1 \times 10^{4} \mathrm{yr}$, which is equal to the cloud crushing time, $t_{\text {crush }}$.

Now let us estimate the radiative cooling timescales. When the SSSF wind impacts the cloud, a double shock structure develops at the contact surface between them, with a forward shock that advances into the cloud with a velocity $v_{\mathrm{s}, \mathrm{c}} \simeq v_{\mathrm{sh}} / q^{0.5}$ and a reverse shock that decelerates the SSSF wind with a velocity $v_{\mathrm{s}, \mathrm{sh}}=v_{\mathrm{sh}}-v_{\mathrm{s}, \mathrm{c}}$. For an SSSF velocity $v_{\mathrm{sh}}=104 \mathrm{~km} \mathrm{~s}^{-1}$ and a density contrast between the cloud and the SSSF of $q=125, v_{\mathrm{s}, \mathrm{c}} \simeq 9.3 \mathrm{~km} \mathrm{~s}^{-1}$ and $v_{\mathrm{s}, \mathrm{sh}} \simeq 95 \mathrm{~km} \mathrm{~s}^{-1}$. The corresponding radiative cooling time of the shocked gas in the cloud, for which the shock velocity is smaller than $80 \mathrm{~km} \mathrm{~s}^{-1}$, can be estimated from Hartigan et al. (1987; see also Gonzalez 2001) as

$$
\begin{aligned}
& t_{\mathrm{c}, \mathrm{c}} \sim 15 \times 10^{4} \mathrm{yr}\left(\frac{v_{\mathrm{s}, \mathrm{c}}}{9.3 \mathrm{~km} \mathrm{~s}^{-1}}\right)^{-3.58} \\
& \times\left(\frac{\rho_{\mathrm{c}}}{1.075 \times 10^{-22} \mathrm{~g} \mathrm{~cm}^{-3}}\right)^{-1}
\end{aligned}
$$

which is larger than the cloud crushing time, a though very sensitive to the value of the shock velocity. On the other hand, the radiative cooling time of the shocked gas behind the SSSF, for which the shock velocity is larger than $80 \mathrm{~km} \mathrm{~s}^{-1}$, is

$$
\begin{aligned}
t_{\mathrm{c}, \mathrm{SSSF}} \sim & 1.8 \times 10^{4} \mathrm{yr}\left(\frac{v_{\mathrm{s}, \mathrm{sh}}}{95 \mathrm{~km} \mathrm{~s}^{-1}}\right)^{1.12} \\
& \times\left(\frac{\rho_{\mathrm{sh}}}{8.6 \times 10^{-25} \mathrm{~g} \mathrm{~cm}^{-3}}\right)^{-1}
\end{aligned}
$$

which is of the order of $t_{\text {crush. As a consequence of this rapid }}$ cooling, the shocked gas of the SSSF wind will produce a cold thin shell around the cloud that will lessen the damage of the impact upon it. The thickness of this cold shell for a shock velocity larger than $80 \mathrm{~km} \mathrm{~s}^{-1}$ is of the order of

$$
d_{\mathrm{c}, \mathrm{SSSF}} \simeq 0.11 r_{\mathrm{c}}\left(\frac{v_{\mathrm{s}, \mathrm{sh}}}{95 \mathrm{~km} \mathrm{~s}^{-1}}\right)^{4.73}\left(\frac{\rho_{\mathrm{sh}}}{8.6 \times 10^{-25} \mathrm{~g} \mathrm{~cm}^{-3}}\right)^{-1}
$$

which is consistent with the thickness of the cold material in the simulations below (see Fig. 4).

The estimates above indicate that since $t_{\mathrm{c}, \mathrm{SSSF}} \sim t_{\mathrm{crush}} \sim$ $2 \tau_{\mathrm{R}-\mathrm{T}} \sim 2 \tau_{\mathrm{K}-\mathrm{H}}$, the radiative cooling will generally play an important role in the evolution of the clouds. Also, as mentioned earlier, a radiative SSSF-cloud interaction will be less
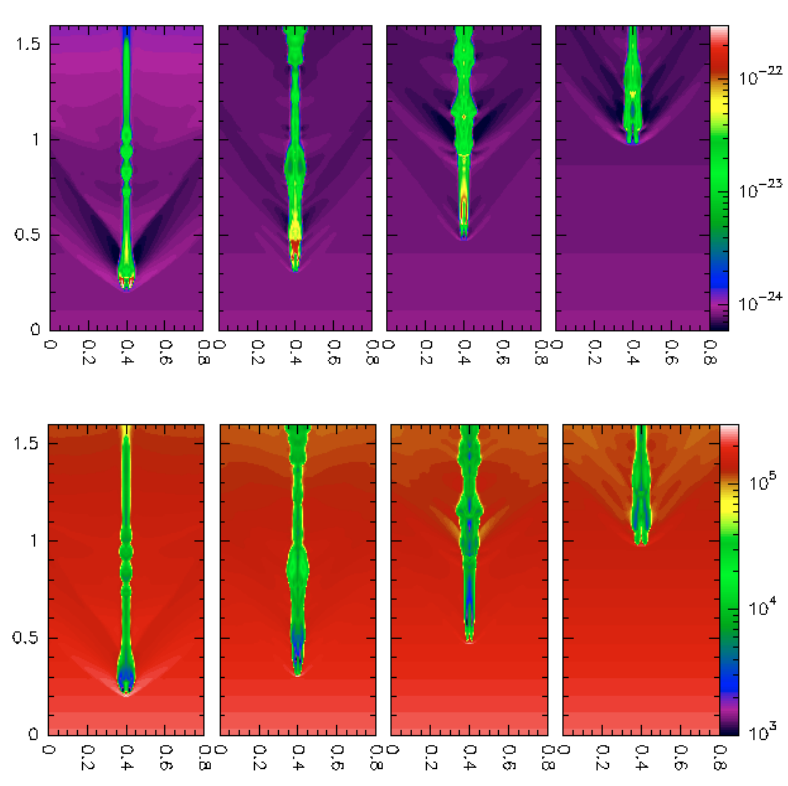

Fig. 4. Model SR1. Color-scale map of the midplane density (top panel) and temperature (bottom panel) distribution (in log scale) for the radiative cooling interaction of a SSSF with one cloud. The initial conditions are the same as in the timescale model of Fig. 3. Time steps are $\left.\left.\left.t=20 t_{\mathrm{SC}} \mathbf{a}\right), t=34 t_{\mathrm{SC}} \mathbf{b}\right), t=50 t_{\mathrm{SC}} \mathbf{c}\right)$, and $\left.t=74 t_{\mathrm{SC}} \mathbf{d}\right)$.

efficient in producing cloud fragmentation than a non-radiative interaction $^{3}$.

Figures 4 to 6 depict this situation. Unlike the results of the non-radiative model SA1, after a radiative cooling interaction a cloud is almost hentirely converted to a long cold, dense filament that has suffered less mass loss to the diffuse ambient gas. As in the non-radiative case, the cloud is essentially compressed and heated by the internal forward shock developed during the impact. This increases the cloud temperature to about $10^{4} \mathrm{~K}$. While the internal shock front compresses the cloud, the external bow shock wave drags the surface cloud material sideways making it lose mass. Therefore, during the time of the interaction, two important effects appear: a continuous mass loss due to the drag of material from the cloud outer parts by the external shock front, and an internal compression and re-expansion that modifies the structure of the cloud core.

In the model SR1, at the last stage of the simulation, the cloud core acquires a normalized velocity $\left(v_{\mathrm{c}, \mathrm{N}}\right.$, Eq. (5)) of 0.34 (that is, $34 \%$ of the SSSF velocity). Similarly in both models SR2 and SR3, the normalized velocities after $50 t_{\mathrm{SC}}$ is 0.4 . Their average velocities over the simulation are $17.3 \mathrm{~km} \mathrm{~s}^{-1}, 20 \mathrm{~km} \mathrm{~s}^{-1}$ and $19.5 \mathrm{~km} \mathrm{~s}^{-1}$, for the models SR1,

\footnotetext{
3 We also notice that before the SSSF wind reaches the clouds, it will impact supersonically the ambient medium at rest thus producing a shock into it that propagates with a velocity $v_{\mathrm{s}, \mathrm{a}} \simeq v_{\mathrm{sh}} /[1+$ $\left.\left(\rho_{\mathrm{a}} / \rho_{\mathrm{sh}}\right)^{0.5}\right] \simeq 69$ (e.g., de Gouveia Dal Pino \& Benz 1993). The shocked ambient material will also suffer relatively rapid cooling, $t_{\mathrm{c}, \mathrm{a}} \sim 2.8 \times 10^{4} \mathrm{yr}\left(v_{\mathrm{s}, \mathrm{a}} / 69 \mathrm{~km} \mathrm{~s}^{-1}\right)^{-3.58}\left(\rho_{\mathrm{c}} / 1.075 \times 10^{-22} \mathrm{~g} \mathrm{~cm}^{-3}\right)^{-1}$. This cold intercloud gas will be pushed along with the SSSF wind and also incorporate the contact discontinuity during the interaction of the SSSF with the clouds.
} 

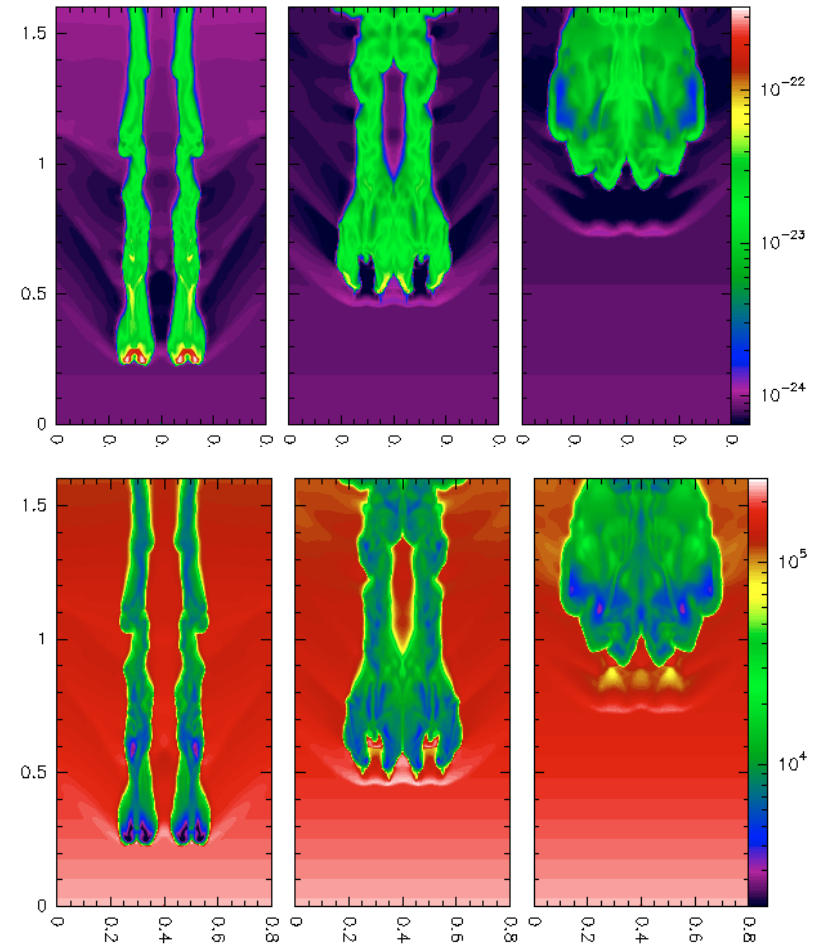

Fig. 5. Model SR2. The same as in Fig. 4, except that here it is considered the radiative cooling interaction between a SSSF and two clouds. Time steps are $\left.\left.t=22 t_{\mathrm{SC}} \mathbf{a}\right), t=36 t_{\mathrm{SC}} \mathbf{b}\right)$, and $t=46 t_{\mathrm{SC}} \mathbf{c}$ ).
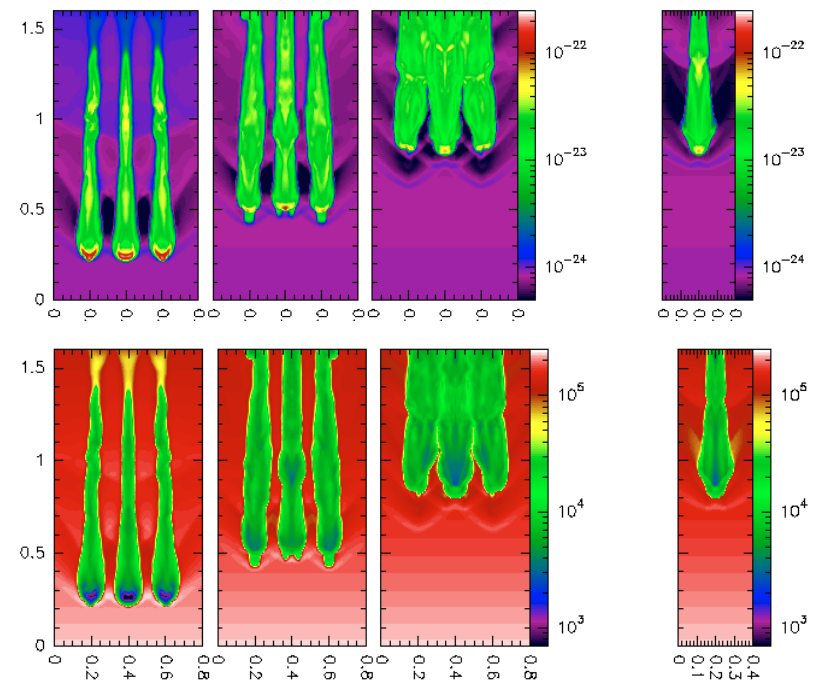

Fig. 6. Model SR3. The same as in Fig. 4, except that here we depict the radiative cooling interaction between an SSSF and three clouds. Time steps are $\left.\left.t=20 t_{\mathrm{SC}} \mathbf{a}\right), t=34 t_{\mathrm{SC}} \mathbf{b}\right)$ and $\left.t=44 t_{\mathrm{SC}} \mathbf{c}\right)$. In the right panel we show the $Y$ - $Z$ midplane density and temperature distribution.

SR2 and SR3, respectively, which are in good agreement, for instance, with cloud velocities inferred from the observations of normal galaxies (see, e.g., Boyce \& Cohen 1994). The evolution of the clouds velocity, shown in Fig. 7, reveals that they tend to accelerate slowly at the beginning of the interaction and faster after $\sim 26 t_{\mathrm{SC}}$, when the core of the cloud has lost mass and is thus accelerated with higher efficiency.

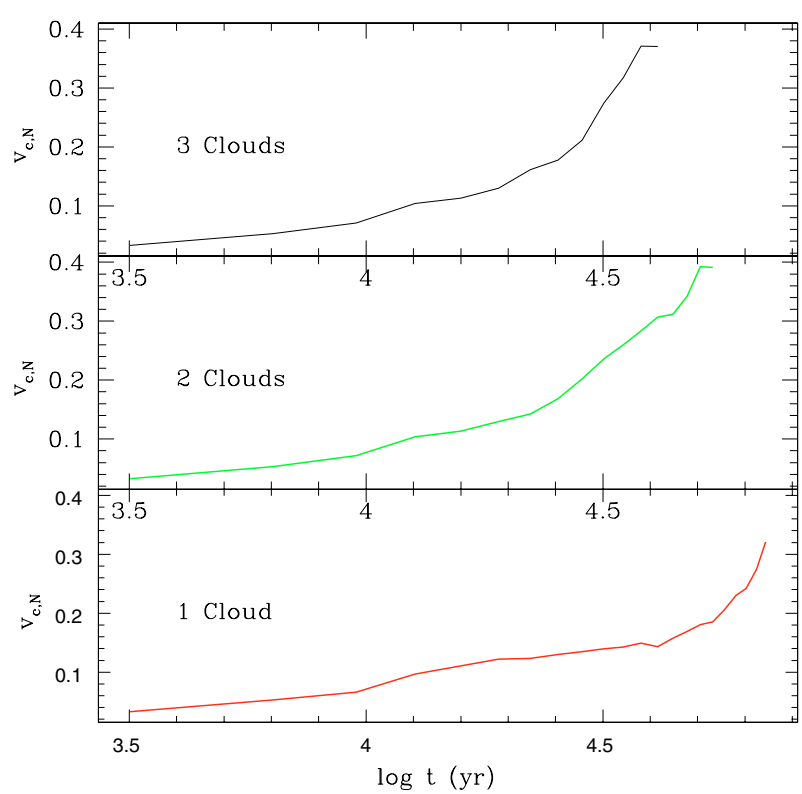

Fig. 7. Velocity evolution of the clouds in the model SR1 (bottom), SR2 (center) and SR3 (top). The velocity is normalized to the SSSF velocity $\left(v_{\mathrm{sh}}=104 \mathrm{~km} \mathrm{~s}^{-1}\right)$ and the time is expressed in logarithmic scale.

The total amount of mass lost by the clouds to the diffuse ambient medium is indicated by the normalized mass loss parameter $M_{1, n}$, defined in Eq. (6). When only one cloud is interacting with the SSSF (SR1), the cloud core is almost completely destroyed at a time $t=50 t_{\mathrm{SC}}$. At $t=20 t_{\mathrm{SC}}$, we obtain $M_{1, n}=0.1$ and at $t=34 t_{\mathrm{SC}}$, we obtain $M_{1, n}=0.6$. At $t=50 t_{\mathrm{SC}}$, from the analysis of the density and the temperature profiles, we find that essentially all the gas that was in the core of the cloud has been ablated by the SSSF.

Klein et al. (1994) find that the maximum time for cloud destruction is $t_{\mathrm{d}, \max } \simeq 3.5\left(r_{\mathrm{c}} q^{0.5} v_{\mathrm{sh}}\right)$. The initial conditions of the models above would imply $t_{\mathrm{d} \text {, max }} \sim 1.8 \times 10^{4} \mathrm{yr}$, that is $\sim 19 t_{\mathrm{SC}}$, but we find that the total mass lost by the cloud in the radiative cooling model SR1 is much less at that time. This indicates a much less efficient cloud destruction as a result of the radiative cooling interaction with the SSSF.

The same occurs in the simulations SR2 and SR3. In the first case, we find $M_{1, n} \sim 0.2$ at $t=23 t_{\mathrm{SC}}$ and $M_{1, n} \sim 0.7$ at $t=36 t_{\mathrm{SC}}$. In the second case, we find $M_{1, n} \sim 0.1$ at $t=20 t_{\mathrm{SC}}$ and $M_{1, n} \sim 0.8$ at $t=34 t_{\mathrm{SC}}$.

At the final stage of the three simulations, the cloud core remnants have become elongated filaments which are colder and denser than the ISM, so that a complete mixing of the cloud material with the ambient gas is not observed. This point is crucial. Due to the radiative cooling even the ablated gas from the clouds does not mix completely with the ISM, but instead remains slightly colder $(T \sim 5000 \mathrm{~K})$ and much denser $\left(\rho \sim 5 \times 10^{-23} \mathrm{~cm}^{-3}\right)$ than the diffuse gas. This means that the re-expansion phase is less efficient here than in the adiabatic case, and the mixing between ISM and cloud gas is prevented or postponed.

We conclude, therefore, that the interaction between the radiative cooling SSSF and the clouds is unable to destroy them 


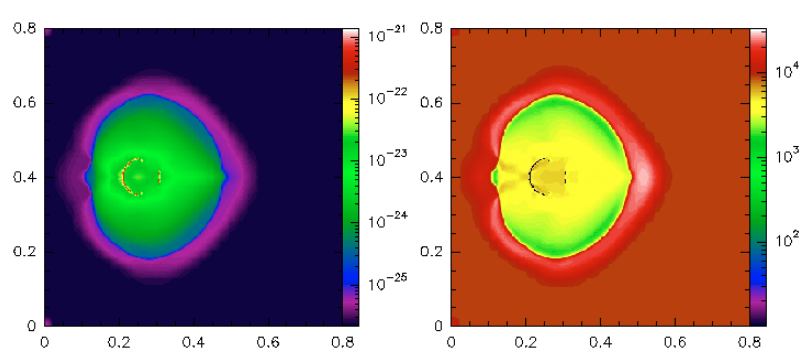

Fig. 8. Model SRP1 before the impact with a SSSF. Maps of the midplane density (left panel) and temperature (right panel) distribution (in $\log$ scale) of a photo-evaporating cloud with $m_{\mathrm{c}} \sim 0.001 M_{\odot}$, $r_{\mathrm{c}}=0.05 \mathrm{pc}$ and $\rho_{\mathrm{c}}=1.075 \times 10^{-22} \mathrm{~g} \mathrm{~cm}^{-3}$. The UV flux is injected from the left of the panel. It has a normalized value $F_{49}=0.2$.

completely. We also find that the number of elongated structures with a density larger than $1 / e$ of the initial cloud density is always the same in the three models above (Figs. 4-6) (i.e., $f_{\mathrm{f}, t}=1$ in Eq. (7)). This means that the cloud core numbers remain unaltered while the outer gas is dragged by the passage of the SSSF.

\subsection{Set 2}

In this section we have run models similar to the previous ones, except that now we have employed the YGUAZUb code, where, besides the radiative cooling, we have also considered the presence of a UV flux of photons. In these simulations, before injecting the SSSF in the computational domain, we have allowed the cloud to be photoionized by the UV flux for $6 \times 10^{3}$ yr. Figure 8 shows a photo-evaporating cloud embedded in a bath of UV photons. The UV flux has been injected from the left, from a star at a distance of $1 \mathrm{pc}$ (that does not lie within the computational domain), with an effective temperature of $60000 \mathrm{~K}$ and an UV flux $F_{49}=0.2$, where $F_{49}$ is the flux in units of $10^{49}$ photons/s.

As we see in Fig. 9, the cloud photoionizes almost completely very rapidly, attaining a temperature in the core of $\sim 10^{4} \mathrm{~K}$. As a consequence, the cloud becomes transparent to the UV photon flux, and the photo-evaporation ceases. At this stage, the only physical phenomenon responsible for the evolution of the cloud is the free thermal expansion. When an SSSF impacts a cloud in such conditions, a forward shock front with a velocity lower than the SSSF but with a higher density develops. This shock front may be seen in Fig. 9 with a mass density $\sim 6.4 \times 10^{-23} \mathrm{~g} \mathrm{~cm}^{-3}$, or 75 times the number density of the SSSF injected at $t=6000 \mathrm{yr}$.

We note that this is substantially different from the case where an SSSF interacts with a neutral photoionizing cloud. In the present analysis the cloud is already fully photoionized when it is impacted by the SSSF, and the UV photons flux that continues to be injected keeps the temperature of the denser structures high enough, thus favoring a faster re-expansion that sweeps away density and temperature fluctuations. For a detailed study of an interaction between a wind and neutral photoevaporating clouds covering an extensive parameter space, we refer to the recent work by Raga et al. (2005).

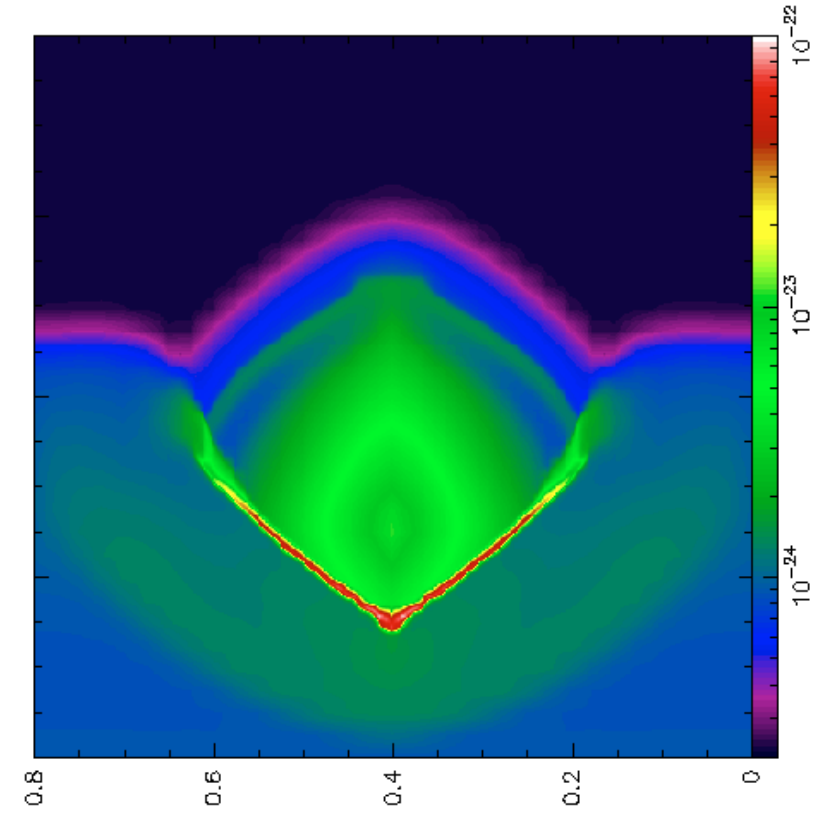

Fig. 9. Density profile (in $\log$ scale) of model SRP1 at a time $t=$ $3.3 t_{\mathrm{SC}}$. The initial conditions are the same as in Fig. 4, except that now the cloud is also under the action of a continuous UV photon flux that comes from the bottom of the box, with $F_{49}=0.2$. The forward shock front resulting from the interaction between the SSSF and the photo-evaporating gas cloud has a mass density of $\sim 6.4 \times 10^{-23} \mathrm{~g} \mathrm{~cm}^{-3}$.

The density and the temperature evolution of models SRP1 and SRP2, with one and two photoevaporated clouds, respectively, are plotted in Figs. 10 and 11. In these cases, the interaction between the forward shock front and the clouds is stronger than in the cases without photo-evaporation, as the effective density contrast between the cloud and the SSSF is only $\sim 1.7$, or 80 times lower than in the models of Set 1 without photoevaporation. As a consequence, the clouds are pushed and expand with higher efficiency. The normalized velocity at a time of $33.7 t_{\mathrm{SC}}$ is 0.5 for the run SRP1 and 0.57 for the run SRP2. These values are higher than those obtained in the runs of Set 1 , and are reached in a shorter time. In Fig. 12, the evolution of the normalized velocity is shown for both cases. Due to the faster acceleration, the resulting average velocity is $36.7 \mathrm{~km} \mathrm{~s}^{-1}$ for the cloud of model SRP1 and $44.5 \mathrm{~km} \mathrm{~s}^{-1}$ for the clouds of model SRP2.

When the SSSF wind is injected, the maximum density of the photo-evaporating clouds is $\sim 2.15 \times 10^{-23} \mathrm{~g} \mathrm{~cm}^{-3}$, that is only $1 / 5$ of the initial cloud density. Taking this value, $\rho_{\mathrm{c}, \mathrm{ph}}$, as the initial density of the photionized cloud at the time of the interaction with the SSSF, we find that the total mass of the core with $\rho \geq \rho_{\mathrm{c}, \mathrm{ph}} / e$ is only $35 \%$ of the initial cloud mass. The rest of the gas spreads in the ISM, with an average mass density of $\sim 2.15 \times 10^{-24} \mathrm{~g} \mathrm{~cm}^{-3}$ and a volume which is sixty times larger than the initial volume. Later, as it interacts with the SSSF, the shocked cloud gas is pushed away and expands more efficiently, but even in this case the mixing with the ISM is not efficient and no significant fragmentation is observed (Figs. 10 and 11). The normalized fragmentation factor (as defined in Eq. (7)) is always 1 for both models SRP1 and SRP2, which is the same found for the models of the set 1 . 

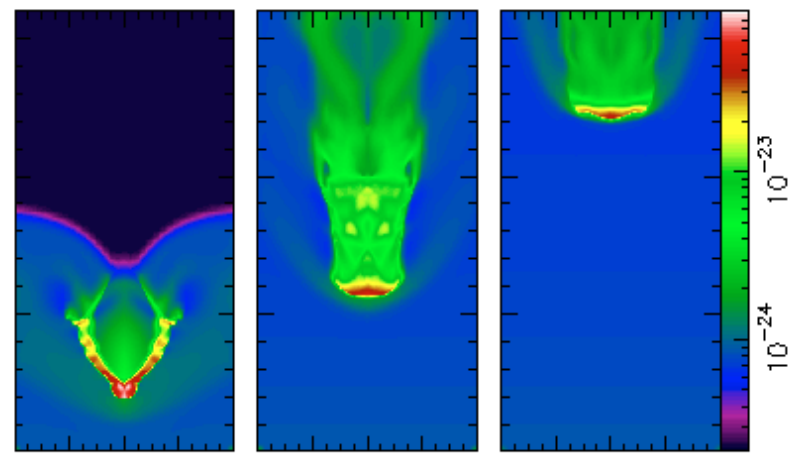

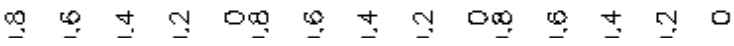
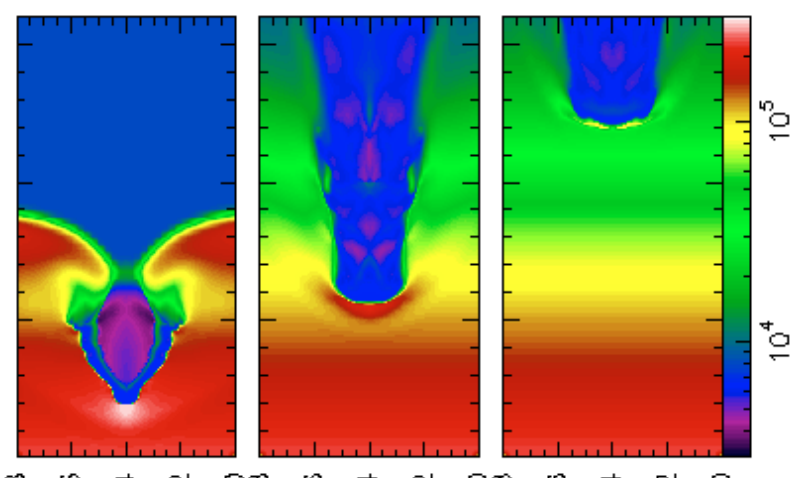

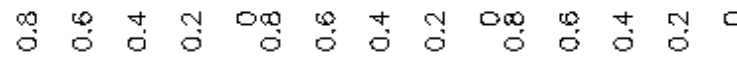

Fig. 10. Later evolution of model SRP1. Color-scale map of the midplane density (top panel) and temperature (bottom panel) distributions (in log scale) for the radiative cooling interaction of an SSSF with a photo-evaporating cloud. The physical conditions are the same as in Fig. 9. Time steps are $\left.\left.t=6.8 t_{\mathrm{SC}} \mathbf{a}\right), t=20.3 t_{\mathrm{SC}} \mathbf{b}\right)$, and $t=33.7 t_{\mathrm{SC}} \mathbf{c}$ ).

\subsection{Set 3}

In the third set of simulations, instead of a steady state shock front (SSSF), we have considered the interaction of a SNR shell with one (models SNS1 and SNSP1) and two clouds (models SNS2 and SNSP2). The simulations without a UV photon flux (SNS1 and SNS2) were run with the YGUAZUa version of the code, and the simulations with a continuous flux of UV photons (SNSP1 and SNSP2) were run with the YGUAZUb code. The results are shown in Figs. 13 to 16.

In this set, we have considered initial conditions appropriate for an SB galaxy environment. Due to the high rate of SN explosions, the ISM is at low density and high temperature, and thus, the SNR shells are expected to have much lower densities than that of the SSSF considered in Sets 1 and 2 (we assume here an SNR shell with a density ten times smaller). The small clouds, presumably formed by the fragmentation of shells formed in an earlier generation of SNe that exploded in the SB ambient (Melioli \& de Gouveia Dal Pino 2004), have a mass density $\rho_{\mathrm{c}}=2.15 \times 10^{-24} \mathrm{~g} \mathrm{~cm}^{-3}$ in the models without photoevaporation, SNS1 and SNS2, and $\rho_{\mathrm{c}}=1.075 \times 10^{-22} \mathrm{~g} \mathrm{~cm}^{-3}$ in the models with photo-evaporation, SNSP1 and SNSP2. This difference between the initial densities of the clouds is justified by the fact that the density contrast between the clouds and the ambient medium is expected to be higher in photoionized regions (see, e.g., Bertoldi \& McKee 1990). In the presence of UV photons, the initial internal pressure of the cloud must be
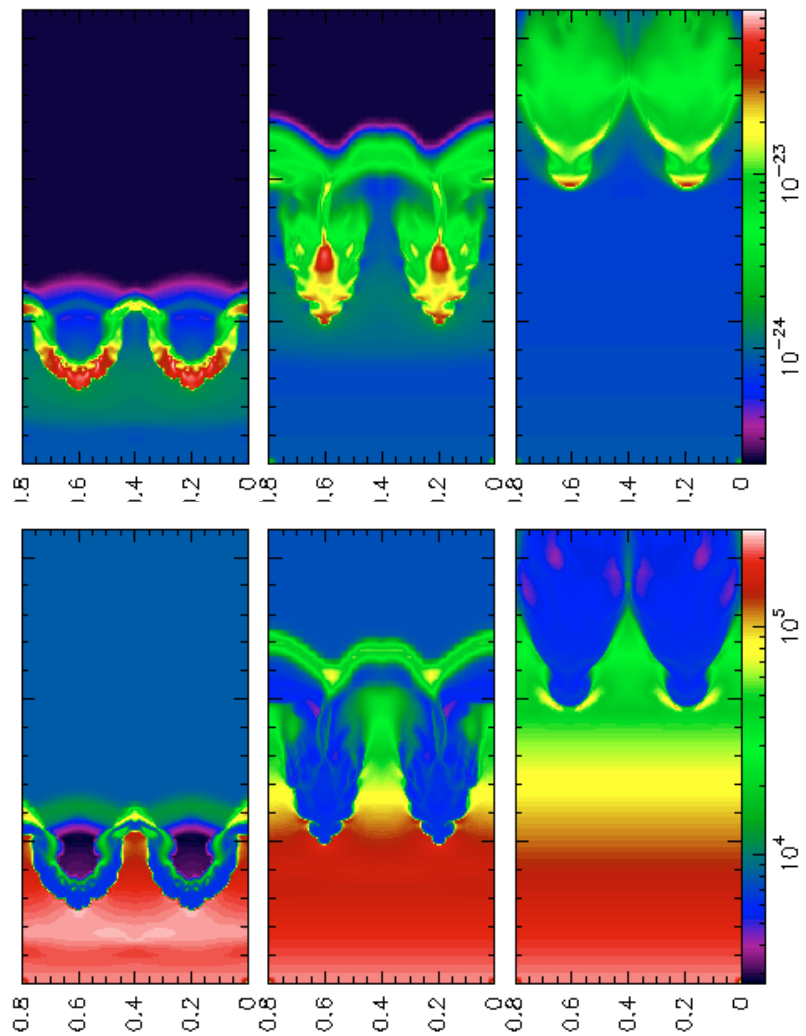

Fig. 11. Model SRP2. The same as in Fig. 9, except that now the radiative cooling interaction between an SSSF and a system of two photo-evaporating clouds is considered. Time steps are $t=6.8 t_{\mathrm{SC}} \mathbf{a}$ ), $\left.t=14 t_{\mathrm{SC}} \mathbf{b}\right)$, and $\left.t=23 t_{\mathrm{SC}} \mathbf{c}\right)$.

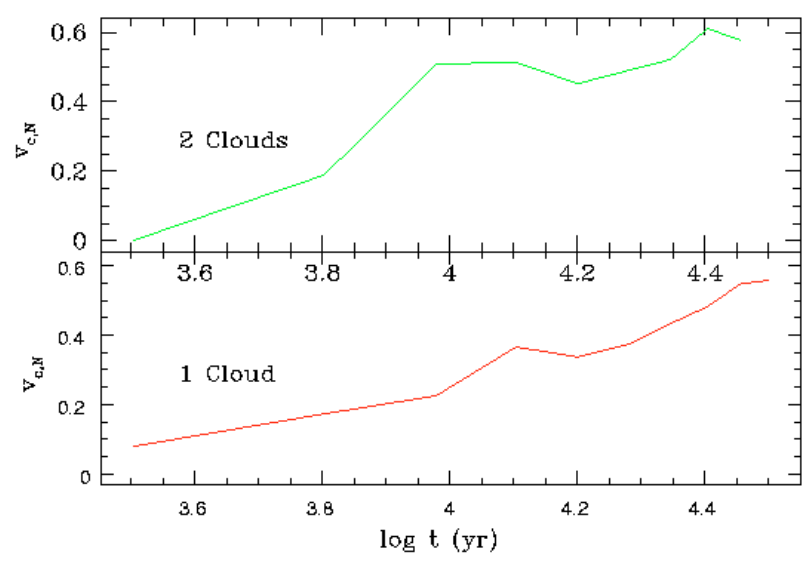

Fig. 12. Velocity evolution of the photoionized clouds of the models SRP1 (bottom), and SRP2 (top). The velocity is normalized to the SSSF velocity, and the time is expressed in logarithmic scale.

higher than the ISM pressure ( $\sim 10$ times) and this forces the expansion of the cloud in the photoionized environment. Thus, for the models SNS1 and SNS2, we take a density contrast five times lower than in the cases studied in the Sets 1 and 2, while for the models SNSP1 and SNSP2, we take a density contrast 10 times higher. The shell is assumed to have a thickness of 1 pc (see, e.g. McCray 1992). Aiming at studying more realistic systems involving SNR-clouds interactions, the parameters we have adopted here such as that the clouds have dimensions that are not much smaller than the thickness of the SNR shell, 

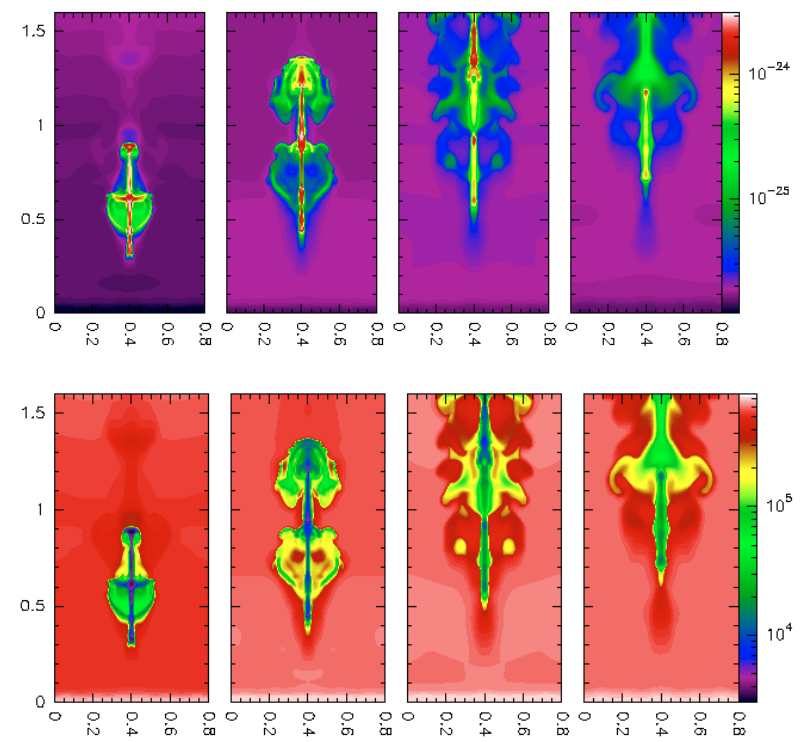

Fig. 13. Model SNS1. Maps of the midplane density (top panel) and temperature (bottom panel) distributions (in log scale) for the interaction of a SNR with one cloud. The cloud has a mass density $\rho_{\mathrm{c}}=2.15 \times 10^{-24} \mathrm{~g} \mathrm{~cm}^{-3}$, a temperature $T=100 \mathrm{~K}$ and a radius $r_{\mathrm{c}}=0.05 \mathrm{pc}$, and is embedded in an ISM which has a mass density $\rho_{\mathrm{a}}=2.15 \times 10^{-26} \mathrm{~g} \mathrm{~cm}^{-3}$ and a temperature $T=10^{4} \mathrm{~K}$. The SNR is injected from the bottom of the box with a velocity of $250 \mathrm{~km} \mathrm{~s}^{-1}$, a mass density $\rho_{\mathrm{sh}}=8.6 \times 10^{-26} \mathrm{~g} \mathrm{~cm}^{-3}$ and a temperature $T=8.2 \times 10^{5} \mathrm{~K}$. Time steps are $\left.\left.\left.t=48 t_{\mathrm{SC}} \mathbf{a}\right), t=81 t_{\mathrm{SC}} \mathbf{b}\right), t=121 t_{\mathrm{SC}} \mathbf{c}\right)$, and $\left.t=162 t_{\mathrm{SC}} \mathbf{d}\right)$.

$r_{\mathrm{c}} \sim h_{\mathrm{sh}} / q^{0.5}$ (see definition of a small cloud by Klein et al. 1994). This justifies in part some of the differences to the results found in the sets 1 and 2, and also explains some differences with respect to analytical solutions, as we will discuss in the next section.

In the cases without photo-evaporation (Figs. 13 and 15), the filamentation of the clouds and gas mixing with the ISM due to the interaction with the SNR is less efficient than in the previous sets with an SSSF, in spite of the smaller density contrast between the cloud and the shell $\left(q=\rho_{\mathrm{c}} / \rho_{\mathrm{sh}}=25\right.$ in Figs. 13 and 15) than in the models of the sets 1 and 2 (where $\left.q=\rho_{\mathrm{c}} / \rho_{\mathrm{sh}}=125\right)$. This is explained by the fact remarked above that in these models of Figs. 13 and 15 (Set 3 ) the thickness of the shock front is not much larger than the size of the clouds. In Figs. 13 and 15, the gas mixing timescale must be much longer than the total computation time which is of the order of $5 \times 10^{5} \mathrm{yr}$.

As in Set 1, we can also estimate the radiative cooling timescales behind the double shock structure that develops at the contact surface between the SNR shell and each cloud after the impact. For a shell velocity $v_{\mathrm{sh}}=250 \mathrm{~km} \mathrm{~s}^{-1}$ and a density contrast $q=25$, we find that the forward shock speed into the cloud is $v_{\mathrm{s}, \mathrm{c}} \simeq 50 \mathrm{~km} \mathrm{~s}^{-1}$ and the reverse shock speed into the SNR shell is $v_{\mathrm{S}, \mathrm{SNR}} \simeq 200 \mathrm{~km} \mathrm{~s}^{-1}$. Thus the radiative cooling time of the shocked gas in the SNR shell is $t_{\mathrm{c}, \mathrm{SNR}} \sim 4.1 \times 10^{5} \mathrm{yr}\left(v_{\mathrm{s}, \mathrm{SNR}} / 200 \mathrm{~km} \mathrm{~s}^{-1}\right)^{1.12}\left(\rho_{\mathrm{SNR}} / 8.6 \times\right.$ $\left.10^{-26} \mathrm{~g} \mathrm{~cm}^{-3}\right)^{-1}$, which is much longer than the cloud crushing time, $t_{\text {crush }} \sim t_{\mathrm{SC}} q^{0.5} \simeq 1.9 \times 10^{3}$ yr. This long cooling
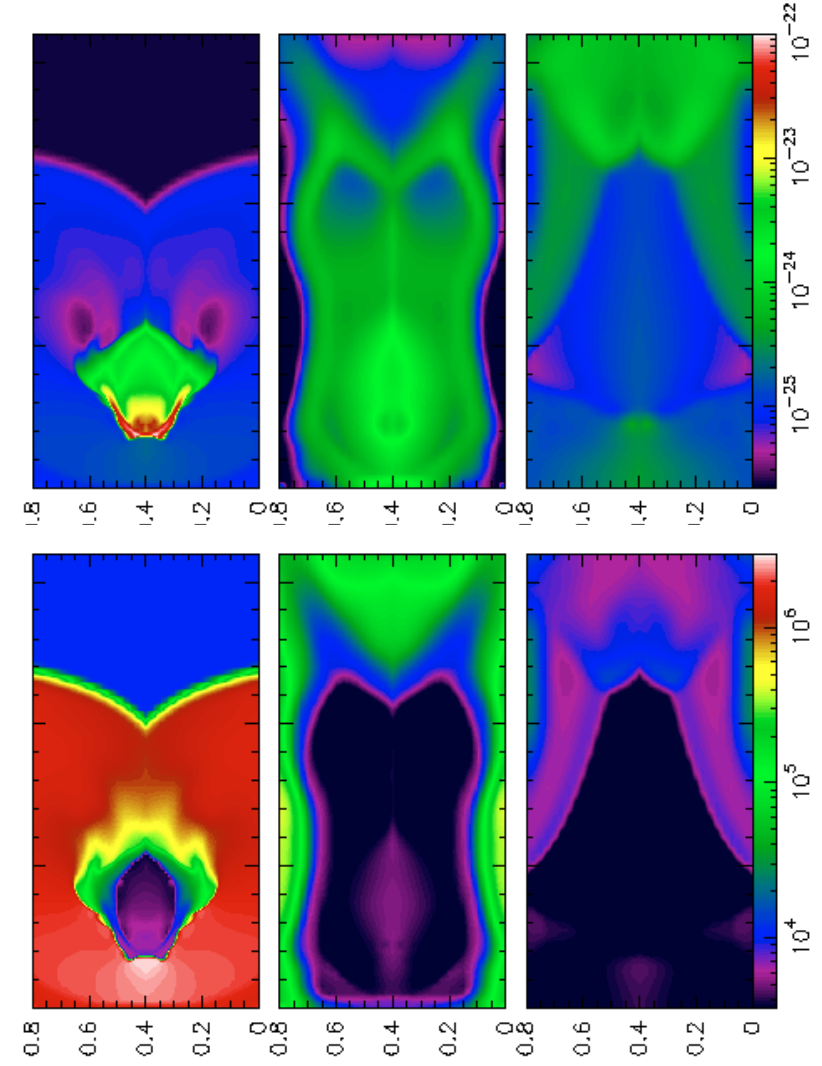

Fig. 14. Model SNSP1. Color-scale map of the midplane density (top panel) and temperature (bottom panel) distributions (in log scale) for the interaction of an SNR with one photo-evaporating cloud. The cloud has a mass density $\rho_{\mathrm{c}}=1.075 \times 10^{-22} \mathrm{~g} \mathrm{~cm}^{-3}$, a temperature $T=100 \mathrm{~K}$ and a radius $r_{\mathrm{c}}=0.05 \mathrm{pc}$ and is embedded in an ISM with a mass density $\rho_{\mathrm{a}}=2.15 \times 10^{-26} \mathrm{~g} \mathrm{~cm}^{-3}$ and a temperature $T=10^{4} \mathrm{~K}$. The SNR is injected from the bottom of the box with a velocity of $250 \mathrm{~km} \mathrm{~s}^{-1}$, a mass density $\rho_{\mathrm{sh}}=8.6 \times 10^{-26} \mathrm{~g} \mathrm{~cm}^{-3}$ and a temperature $T=8.2 \times 10^{5} \mathrm{~K}$. Time steps are $\left.\left.t=8.1 \times 10^{3} \mathrm{yr} \mathbf{a}\right), t=48.7 t_{\mathrm{SC}} \mathbf{b}\right)$, and $\left.t=129 t_{\mathrm{SC}} \mathbf{c}\right)$.

time is due to the very low density of the shell material. An even longer radiative cooling time is found for the shocked ambient material $\left(\sim 1 \times 10^{6} \mathrm{yr}\right)$ which is more rarified than the shell. On the other hand, the radiative cooling time of the shocked material in the cloud is much smaller, $t_{\mathrm{c}, \mathrm{c}} \sim$ $1.8 \times 10^{4} \mathrm{yr}\left(v_{\mathrm{s}, \mathrm{c}} / 50 \mathrm{~km} \mathrm{~s}^{-1}\right)^{-3.58}\left(\rho_{\mathrm{c}} / 2.15 \times 10^{-24} \mathrm{~g} \mathrm{~cm}^{-3}\right)^{-1} \simeq$ $9 t_{\text {crush }} \simeq 47 t_{\mathrm{SC}}$. A though longer than the cloud crushing time, this time scale is short enough to provide the observed radiative cooling of the shocked cloud material in the models of Figs. 13 and 15 within a few crushing times. We can also estimate the thickness of the cold shell that develops due to the cooling of the shocked material around each cloud. For a shock velocity smaller than $80 \mathrm{~km} \mathrm{~s}^{-1}$ (Hartigan et al. 1987), $d_{\mathrm{c}, \mathrm{c}} \simeq 0.3 r_{\mathrm{c}}\left(v_{\mathrm{s}, \mathrm{c}} / 50 \mathrm{~km} \mathrm{~s}^{-1}\right)^{-4.51}\left(\rho_{\mathrm{c}} / 2.15 \times 10^{-24} \mathrm{~g} \mathrm{~cm}^{-3}\right)^{-1}$, which is compatible with the thickness of the cold material in the simulations (see Figs. 13 and 15).

The clouds in Figs. 13 and 15 are less accelerated by the shock front than in the sets with an SSSF and develop a very elongated filamentary structure with a temperature of $\sim 5 \times 10^{3} \mathrm{~K}$ and a density of $\sim 2.15 \times 10^{-24} \mathrm{~g} \mathrm{~cm}^{-3}$ that grows with time. The normalized velocity of the elongated filaments 


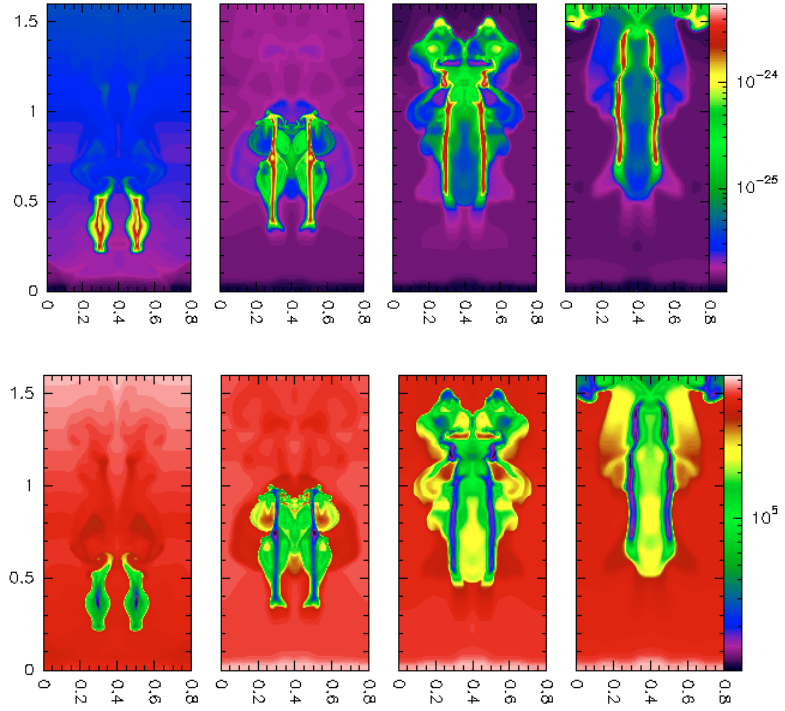

Fig. 15. Model SNS2. The same as in Fig. 15, except that now the radiative cooling interaction between an SNR and two clouds is considered. Time steps are $\left.\left.\left.t=48 t_{\mathrm{SC}} \mathbf{a}\right), t=89 t_{\mathrm{SC}} \mathbf{b}\right), t=129 t_{\mathrm{SC}} \mathbf{c}\right)$ and $\left.t=162 t_{\mathrm{SC}} \mathbf{d}\right)$.

are only 0.04 after a time of $129 t_{\mathrm{SC}}$ for both models SNS1 and SNS2. The evolution of the cloud velocity is shown in Fig. 17. For model SNS1, after $162 t_{\mathrm{SC}}$ (the total simulated interval), we obtain a normalized mass loss to the ISM of only $M_{1, n}=0.5$, so that half of the mass of the cloud is still in the filaments. For model SNS2, we obtain, after $56 t_{\mathrm{SC}}, M_{1, n}=0.2$, and after $162 t_{\mathrm{SC}}, M_{1, n}=0.8$. In these cases no significant fragmentation of the elongated structures is produced during the simulations. In these cases, the elongated filaments have a thickness of $\sim 0.03 \mathrm{pc}$; as we have seen in Eq. (9), the most disruptive Kelvin-Helmholtz (K-H) modes have wavelengths corresponding to $k r_{\mathrm{c}} \simeq 1$. If we substitute $r_{\mathrm{c}}$ by the half-thickness of the filament, we obtain that the most disruptive wavelength is $\lambda=2 \pi / k \sim 0.1 \mathrm{pc}$ which corresponds to 64 grid points. This indicates that the absence of $\mathrm{K}-\mathrm{H}$ fragmentation is not due to unresolved physics of our grid but to the presence of radiative cooling as discussed earlier.

When a UV photon flux is present, the results change considerably (Figs. 14 and 16). When the SNR shell is injected, the cloud core has already lost $50 \%$ of its initial mass due to photoevaporation, and the forward shock wave that is produced from the interaction of the SNR with this photo-evaporating cloud is more efficient in destroying it. The interaction accelerates the cloud to a normalized velocity of 0.08 of the SNR shell velocity, and since all the cloud is at a temperature greater than $5000 \mathrm{~K}$, the shocked gas begins to expand and fill in the environment around the cloud. No fragments are observed, and the cloud cores rapidly disappear in both Figs. 14 and 16. In this situation, a real mixing between the ISM and the cloud gas takes place very rapidly in the SB environment. In a time of $129 t_{\mathrm{SC}}$, that is $\sim 5 \times 10^{4} \mathrm{yr}$, the ambient medium increases its mass density to a value of $2.15-6.4 \times 10^{-25} \mathrm{~g} \mathrm{~cm}^{-3}$. This is 10 times greater than the initial ISM density, while the ISM temperature remains of the order of $10^{3}-10^{4} \mathrm{~K}$. This result is
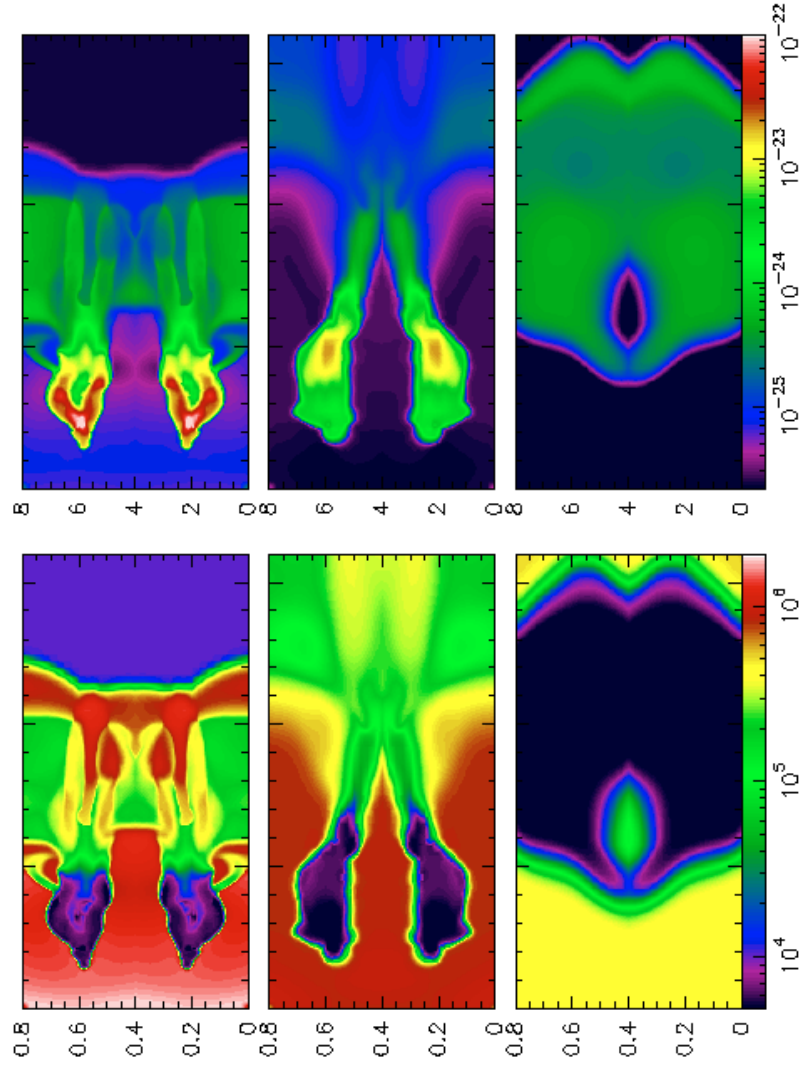

Fig. 16. Model SNSP2. The same as in Fig. 16, except that now the radiative cooling interaction between an SNR and two photoevaporating clouds is considered. Time steps are $t=6.5 \times 10^{3} \mathrm{yr} \mathbf{a}$ ), $\left.\left.t=13 t_{\mathrm{SC}} \mathbf{b}\right), t=27.6 t_{\mathrm{SC}} \mathbf{c}\right)$ and $\left.t=97.4 t_{\mathrm{SC}} \mathbf{d}\right)$.

consistent with that obtained in previous work by Melioli \& de Gouveia Dal Pino (2004) where the timescale for mixing of the gas from the clouds with the ISM in a SB environment due to photo-evaporation was found to be much smaller $\left(\sim 10^{4} \mathrm{yr}\right)$ than the mixing timescale due to mass loss from SNR-cloud interactions.

\section{Discussion and conclusions}

\subsection{Cloud velocity}

When a cloud is impacted, its equation of motion has the approximate form:

$m_{\mathrm{c}} \frac{\mathrm{d} v_{\mathrm{c}}}{\mathrm{d} t} \sim v_{\mathrm{sh}}^{2} \Sigma_{\mathrm{c}} \rho_{\mathrm{sh}}$

where $\Sigma_{\mathrm{c}}$ is the cloud cross section, $m_{\mathrm{c}}$ is the cloud mass and $\rho_{\mathrm{sh}}$ is the density of the shell or wind that impacts on it. Thus, from Eq. (5), the normalized velocity is:

$v_{\mathrm{c}, \mathrm{N}} \sim \frac{v_{\mathrm{sh}} \Sigma_{\mathrm{c}} \rho_{\mathrm{sh}} t}{m_{\mathrm{c}}}$

where we have assumed that the cloud mass and cross section and the shock wave density do not change with time. If we substitute the initial values adopted in our simulations, the normalized velocity of the clouds is $v_{\mathrm{c}, \mathrm{N}} \sim 10^{-5} t$ for both sets 1 and 2, 


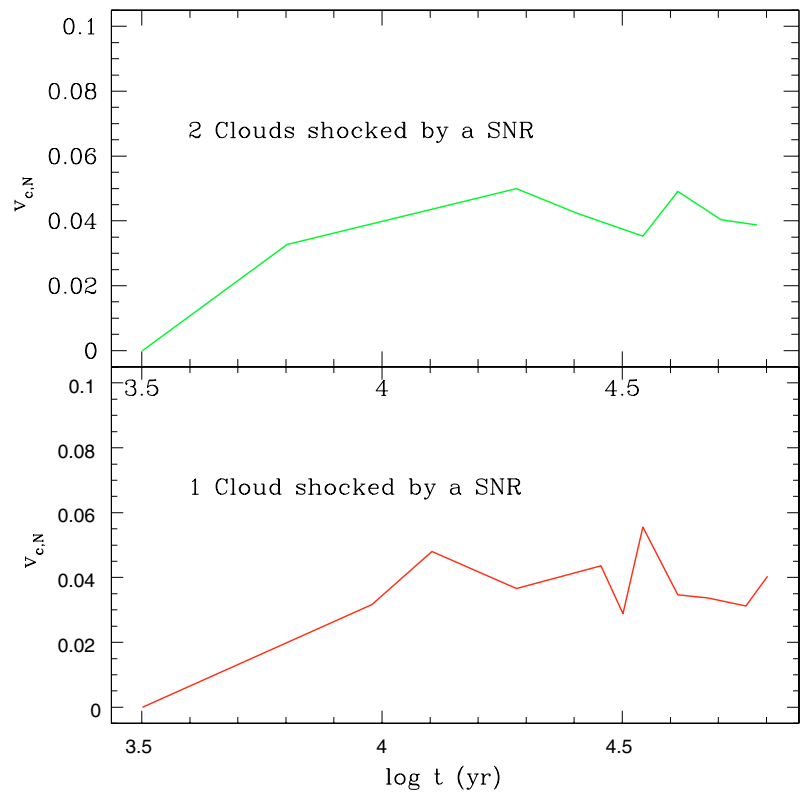

Fig. 17. Velocity evolution of the models SNS1 (bottom), and SNS2 (top). The velocity is normalized to the SNR shell velocity, and the time is expressed in logarithmic scale.

and $v_{\mathrm{c}, \mathrm{N}} \sim 1.5 \times 10^{-4} t$ for set 3 , where the time is expressed in years.

This means that after a time of $\sim 5 \times 10^{4} \mathrm{yr}$ the cloud should have a velocity $\sim 0.5$ times the shock wave velocity in the case of sets 1 and 2 that involve interactions of clouds with an SSSF, which is in agreement with the value inferred from the numerical simulations, while for the simulations of set 3 , where the interaction is with a thin SNR shell, the inferred normalized velocity from the simulations is 100 times smaller than that expected from Eq. (14).

The velocity evolution of a cloud depends on the density, mass and size of the cloud, and on the velocity and density of the shock wave. We have seen that in the presence of radiative cooling, the cloud fragmentation and destruction is inhibited and thus we should expect a larger effective cloud interacting surface with the shock front than in a non-radiative case which would tend to increase the final cloud velocity. With the values above for the velocity, we find that the maximum displacement $\left(L_{\mathrm{M}}\right)$ that a cloud can undergo before being destroyed, in units of the cloud radius, is:

$L_{\mathrm{M}} \sim 2 \psi v_{\mathrm{c}, \mathrm{N}} r_{\mathrm{c}}(\mathrm{pc})$

where $\psi$ is:

$\psi=\frac{t_{\mathrm{des}}}{t_{\mathrm{SC}}}$

and $t_{\mathrm{des}}$ is the destruction time of the cloud. From the results shown above, we find that in all radiative cooling simulations $\psi \geq 100$. Thus, the maximum displacement of the cloud is greater than the value obtained by PFB02, of the order of $3.5 r_{\mathrm{c}}$, in their study of SSSF-clouds interactions, which corresponds to $\psi \sim 10$. We can thus conclude that radiative cooling interactions postpone the destruction of the clouds and increase their velocity. When shocked by an SNR, Eq. (15) indicates that they can be displaced over distances of $\sim 10-20$ times the cloud radius (for $v_{\mathrm{c}, \mathrm{N}} \sim 0.5$ ).

\subsection{Mass loading}

When a cloud is shocked either by an SSSF wind or an SNR, its gas is compressed by a forward shock wave, as discussed in Sect. 2. After this phase, the compressed gas begins to expand into the ISM and part of the gas is also ablated by the external shock wave. This causes an increase of the cloud volume and a decrease of its density. Theoretically, this phenomenon could be responsible for the mixing of the cloud gas with the ISM and contribute to the global increase of the ISM density due to the interaction between several clouds and SNRs. However, the simulations above without photo-evaporation have shown that the forward shock that propagates into the clouds is not able to effectively destroy them. The situation is apparently different in the case where photo-evaporation is also considered. In this case, especially in the runs SNSP1 and SNSP2, the volume filling factor increases to values greater than 1000 times the initial volume of the cloud. This means that the gas of the cloud is really mixed with the ISM and that the mass loading is very efficient. However, this result is caused mainly by the photo-evaporation of the clouds that expand very fast occupying all the ambient medium. From these simulations, which include the processes of photo-evaporation, ablation and the passage of the shock wave through the cloud, we infer that the clouds are destroyed in at least $20000 \mathrm{yr}$, and the corresponding mass loss rate (in terms of the total cloud mass) is $\dot{m}_{\mathrm{c}} \sim 5 \times 10^{-5} m_{\mathrm{c}} M_{\odot} \mathrm{yr}^{-14}$. This is in agreement with the results obtained by Melioli \& de Gouveia Dal Pino (2004), where it has been shown that the combined effects of ablation and photo-evaporation of the clouds can lead to an increase of the ISM density in SB environments.

Now, returning to the cases without photo-evaporation, where we have only considered the radiative cooling interaction of the clouds with a SNR (as in the models SNS1 and SNS2), the results are substantially different. A thin cold dense shell develops at the contact discontinuity between them which is formed from the radiative cooling of the cloud shocked material (see Sect. 3). In this case, the cloud gas is much less efficiently mixed with the ISM, and the only important effect resulting from the interaction is the formation of dense cold filaments with densities up to $\sim 100$ times larger than that of the ambient medium and temperatures up to $\sim 50$ times smaller than that of the ambient medium. These structures are observed in several SBs and galactic winds (see, e.g., Cecil et al. 2001), and do not contribute to increase the density of the diffuse ambient medium (where the observed temperature and density flutuations are, in general, not greater than a factor $\sim 2$ ). Comparing our results with those obtained by PFB02, it is possible to highlight the fact that in radiative cooling interactions the gas mixing is reduced and postponed to much later times. In PFB02, after a time of $68 t_{\mathrm{SC}}$, the gas of each cloud has almost completely mixed with that of the other clouds and

${ }^{4}$ For strong shocks, the typical timescale for the destruction of the cloud is mainly dependent on the cloud mass. 
with the ISM; at this time the clouds have lost their identity and the shock front has destroyed all the denser structures. In our study, on the other hand, the clouds become denser, colder filaments and only the gas of their external parts is dragged by the SNR shell. In the presence of a flux of UV photons, the photoevaporation is the main mechanism responsible for most of the mass loss of the clouds to the ambient medium, therefore confirming the results of Melioli \& de Gouveia Dal Pino (2004). This means that radiative cooling SNR-cloud interactions are unable to destroy the clouds, especially in an SB environment where there are small, dense clouds and an ISM with low density. Instead, these interactions produce cold filaments and do not contribute to significantly increase the ambient density.

\subsection{Fragmentation and destruction of the clouds}

We determine the lifetime of a shocked cloud and its mass evolution. Let us consider the cases of cloud interactions with an SSSF. From the study above we can see that the radiative cooling clouds do not suffer significant fragmentation during the interaction, and the gas is not efficiently mixed with the rest of the ISM. The main point is to understand why fragmentation does not occur. We may distinguish two aspects. First, both the shape and the mass of the cloud change with time. Second, the perturbations in the cloud gas are generally dissipated faster than in the adiabatic case. The resulting elongated dense filaments still belong to the original cloud structure and are therefore separated from the diffuse gas phase of the ISM. This result is a consequence of the fact that all the gas of the cloud is impacted approximately with the same intensity and in the same direction (since $r_{\mathrm{c}}<h_{\mathrm{sh}}$ ), and that the radiative cooling dissipates most of the small perturbations, preventing their increase with time. The shock wave may be unable to destroy the cloud, and other physical phenomena, like ablation by the ISM, thermal conduction and photo-evaporation, are necessary to have efficient mixing (e.g., Melioli \& de Gouveia Dal Pino 2004). Certainly, the shocked gas of the cloud tends to expand because of the increase of its internal pressure, but this expansion is opposed by the high external pressure of the shocked ambient medium, as we can see in Fig. 18, where the profile of the pressure of the model SNS1 is shown at a time $81 t_{\mathrm{SC}}$. The efficient radiative cooling of the shocked gas inside the cloud inhibits its destruction and mixing with the ISM.

Thus, the interaction between one (or more) cloud(s) and a shock wave may be considered as a sum of two effects: an ablation caused by the drag and a re-expansion of the cloud core generated by the passage of an internal forward shock wave. Taking into account the typical mass loss rate from drag (Klein et al. 1994), our cloud should be destroyed by the drag in at least $18000 \mathrm{yr}$ only, which is much shorter than the time we observe in our simulations, that is $\sim 36000 \mathrm{yr}$. This discrepancy increases if we consider that in the study of PFB02 the clouds are destroyed in $\sim 10000 \mathrm{yr}$.

The results obtained in this work show that the gas of clouds is not ablated with the efficiency predicted in previous studies that did not take into account the effects of radiative cooling and photo-evaporation, and that SNR-cloud

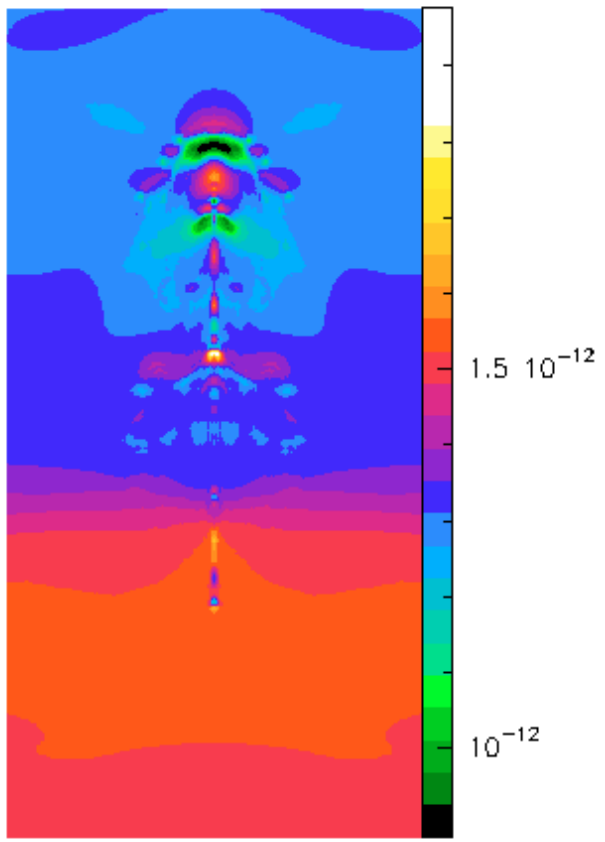

Fig. 18. Model SSN1: pressure profile (in log scale) at a time $t=81 t_{\mathrm{sh}}$.

interactions only are unable to guarantee an efficient mixing of the cloud gas with the ISM. A single cloud shocked by an SSSF (model SR1) suffers a considerable lengthening in the direction of the passage of the SSSF and also in the case of the interaction with a SNR (model SNS1), and the resulting elongated filamentary cloud moves without exchanging much mass with the ISM. In the case of two or three clouds, the mixing is greater, but the elongated structures are still present. A comparison of these results with those obtained by PFB02 indicates that the radiative cooling plays a fundamental role in the evolution of the clouds; they are more accelerated, only partially fragmented, and its gas is spread in the ISM with a lower efficiency, so that no global increase of the ambient density is observed due to these interactions. Only in the presence of UV photon flux is it possible for the clouds to undergo an efficient mass loss to the ISM, therefore producing significant variations in the density, temperature and chemical composition of the ISM. In this case the combination of photo-evaporation, ablation and the shock propagation (which causes cloud expansion due to heating of the gas) determines the growth of the ambient density by a factor of 10 with respect to the initial density.

These simulations have been performed with initially small clouds, $r_{\mathrm{c}}<h_{\mathrm{sh}}$. When the cloud size is comparable to or larger than the SNR shell width, the evolution of the cloud is expected to be very different (see, e.g., Fragile et al. 2004), and instead of gas mixing we may expect an increase of the cloud density itself over a timescale sufficiently large to produce cloud collapse and, perhaps, a process of star formation. This more focused study on processes of star formation is in progress (Melioli et al., in preparation).

Acknowledgements. C.M. and E.M.G.D.P acknowledge financial support from the Brazilian Agencies FAPESP and CNPq. The authors also acknowledge the careful revision and the useful suggestions of the anonymous referee that have helped to improve this work. 


\section{References}

Anderson, M. C., Jones, T. W., Rudnick, L., Tregillis, I. L., \& Kang, H. 1994, ApJ, 421, L31

Begelman, M. C., \& McKee, C. F. 1990, ApJ, 358, 375

Bertoldi, F. 1989, ApJ, 346, 735

Bertoldi, F., \& McKee, C. F. 1990, ApJ, 354, 529

Blondin, J. M., Fryxell, B. A., \& Konigl, A. 1990, ApJ, 360, 370

Boyce, P. J., \& Cohen, R. J. 1994, A\&AS, 107, 563

Cecil, G., Bland-Hawthorn, J., Veilleux, S., \& Fillipenko, A. V. 2001, ApJ, 555, 338

Cerqueira, A. H., \& de Gouveia dal Pino, E. M. 1999, ApJ, 510, 828

Chandrasekhar, S. 1961, Hydrodynamic and Hidromagnetic Stability (New York: Dover)

Chevalier, R. A. 1974, ApJ, 188, 501S

Cioffi, D. F., \& Shull, J. M. 1991, ApJ, 367, 96

Colina, L., Lipari, S., \& Macchetto, F. D. 1991, ApJ, 379, 113

de Gouveia Dal Pino, E. M. 1999, ApJ, 526, 862

de Gouveia dal Pino, E. M., \& Benz, W. 1993, ApJ, 410, 686

Fragile, P. C., Murray, S. D., Anninos, P., \& van Breugel W. 2004, ApJ, 604, 74

Fragile, P. C., Anninos, P., Gustafson, K., \& Murray, S. D. 2005, ApJ, 619,327

Gonzalez, R. D. 2001, Ph.D. Thesis, Universidad Autonoma de Mexico

Gorti, U., \& Hollenbach, D. 2002, ApJ, 573, 215

Hartigan, P., Raymond, J., \& Hartmann, L. 1987, ApJ, 316, 323

Hartquist, T. W., Dyson, J. E., Pettini, M., \& Smith, L. J. 1986, MNRAS, 220, 801

Jun Byung-Il, \& Jones, T. W. 1999, ApJ, 511, 774

Jun, B. I., Jones, T. W., \& Norman, M. L. 1996, ApJ, 468, L59
Klein, R. I., McKee, C. F., \& Colella, P. 1994, ApJ, 420, 213

Lim, A. J., \& Raga, A. C. 1999, MNRAS, 303, 546

Marcolini, A., Strickland, D. K., D’Ercole, A., Heckman, T. M., \& Hoopes, C. G. 2005 [arXiv: astro-ph/0506645]

Masciadri, E., de Gouveia Dal Pino, E. M., Raga, A. C., \& Noriega-Crespo, A. 2002, ApJ, 580, 950

Melioli, C., \& de Gouveia Dal Pino, E. M. 2004, A\&A, 424, 817

McKee, C. F. 1988, in Supernova Remnants and the Interstellar Medium, ed. R. S. Roger, \& T. L. Landecker (Cambridge: University Press), IAU Coll., 101, 205

McKee, C. F., \& Begelman, M. C. 1990, ApJ, 358, 392

Nittmann, J., Falle, S. A. E. G., \& Gaskell, P. H. 1982, MNRAS, 201, 833

Ott, J., Martin, C. L., \& Walter, F. 2003, ApJ, 594, 776

Poludnenko, A. Y., Frank, A., \& Blackman, E. G. 2002, ApJ, 576, 832

Raga, A. C., de Gouveia Dal Pino, E. M., Noriega-Crespo, A., Minnini, P. D., \& Velázquez, P. F. 2002, A\&A, 392, 267

Raga, A. C., Navarro-Gonzalez, R., \& Villagran-Muniz, M. 2000, Rev. Mex. Astron. Astrofis., 36, 67

Raga, A. C., \& Reipurth, B. 2004, Rev. Mex. Astron. Astrofis., 40, 15

Raga, A. C., Steffen, W., \& González, R. F. 2005, Rev. Mex. Astron. Astrofis., 41, 45

Redman, M. P., Williams, R. J. R., \& Dyson, J. E. 1998, MNRAS, 298, 33

Scoville, N., \& Soifer, B. T. 1990, in Massive stars and starburst, ed. C. Leitherer, N. R. Walborn, T. M. Heckman, \& C. A. Norman, 233, 238

Steffen, W., \& Lopez, J. A. 2004, ApJ, 612, 319

Stone, J. M., Xu, J., \& Hardee, P. E. 1997, ApJ, 483, 121

Van Leer, B. 1982, ICASE, Report No. 82-30

Young, T. R., \& Boris, J. P. 1973, NRL Mem. REep. 2611 